\title{
NÚCLEOS DE POBLACIÓN EN RIESGO DE INUNDACIÓN FLUVIAL EN ARAGÓN: DIAGNÓSTICO Y EVALUACIÓN PARA LA ORDENACIÓN DEL TERRITORIO*
}

\author{
Sergio Doménech Zueco, Alfredo Ollero Ojeda y Miguel Sánchez Fabre \\ Dpto. de Geografía y Ordenación del Territorio, Facultad de Filosofía y Letras \\ Universidad de Zaragoza \\ sdome@unizar.es
}

\begin{abstract}
Resumen: Se presenta un inventario de núcleos de población que se encuentran en situación de riesgo por inundaciones fluviales en Aragón. La identificación y diagnóstico de estos núcleos se ha realizado mediante fotointerpretación. Los casos de estudio se han catalogado en función del tipo de curso fluvial que los atraviesa y la densidad de poblamiento que puede verse afectada en las inundaciones. La información se ha analizado por provincias, por tipología de cursos fluviales y por la densidad de edificaciones. La evaluación de los núcleos en riesgo se ha realizado con evidencias de crecidas históricas, actuaciones en cauces que minimicen la peligrosidad y la inclusión del núcleo en el Plan Especial de Protección Civil ante inundaciones en la Comunidad Autónoma de Aragón. Esta evaluación preliminar del riesgo debe ser útil para la ordenación del territorio a escala autonómica por lo que se proponen líneas de actuación para el futuro.
\end{abstract}

Palabras clave: riesgos naturales, inundaciones fluviales, núcleos de población, ordenación del territorio, Aragón.

\begin{abstract}
This paper gathers an inventory of population settlements that are in situation of risk by fluvial floods in Aragón. The diagnosis of these nucleus has been made by means of photo-interpretation. The cases of study have been catalogued based on the type of fluvial course that crosses them and the density of settlement that can be affected by the floods. The information has been analyzed according to provinces, by the kind of fluvial course and by the density of constructions. The assessment of the population settlements has been completed with the existence of past floods, the interventions in rivers that minimizes the hazard probability and their inclusion in the Flood Special Plan in
\end{abstract}

* Recibido: 2-9-08. Aceptado: 26-01-09. 
Aragón made by Civil Protection. This assessment can be useful for land management at regional scale, and for this reason lines of action for the future are set out.

Key words: natural hazards, river floods, population settlements, land management, Aragón

\section{Introducción}

Las crecidas fluviales son episodios extremos necesarios para el correcto funcionamiento de los ríos y de sus ecosistemas asociados. Sin embargo, las sociedades ribereñas no conviven bien con las avenidas, sino que cada vez sufren mayores daños al tener más intereses en los espacios inundables. En países desarrollados hay en la actualidad suficientes mecanismos de planificación y sistemas de seguridad como para que una crecida no sea un drama, sino un acontecimiento beneficioso para el río y para la propia educación ciudadana.

Para el presente trabajo se ha elegido como área de estudio la Comunidad Autónoma de Aragón, que presenta una gran variedad hidromorfológica de cursos fluviales, desde el Ebro, el más caudaloso que drena el territorio, hasta pequeñas ramblas y barrancos de caudal intermitente (Del Valle et al., 2007), pasando por una contrastada gama de longitudes y caudales en las tres grandes subcuencas hidrográficas que drenan el territorio: la del Ebro (al centro y norte de la comunidad), la del Júcar (al Sur) y la del Tajo (al suroeste en muy pequeña proporción). En las dos primeras demarcaciones se incluye el 99,8\% (88 y 11,8\% respectivamente) del territorio aragonés (Sánchez Fabre et al., 2004). Las inundaciones constituyen el riesgo natural más extendido en el territorio aragonés, muy por delante de otros tanto en frecuencia de episodios como en daños generados (Pujadas, 2002). La variada red hidrográfica aragonesa ha ofrecido episodios de crecidas fluviales con dinámicas muy diferentes que han generado en numerosos casos inundaciones en los núcleos urbanos que atraviesan. En este trabajo sólo se estudian las inundaciones fluviales, derivadas del desbordamiento de los cauces en procesos de crecida.

Las inundaciones fluviales que se dan en Aragón responden a variados procesos de crecida, muy difíciles de clasificar, pero que Ollero $(2000,2006)$ simplificó en tres grandes grupos:

a) Procesos en cursos de montaña y cauces de orden bajo (pequeños valles pirenaicos, barrancos y ramblas de la Depresión del Ebro y de la Cordillera Ibérica) que se manifiestan generalmente en crecidas relámpago (flash-flood), bruscas y rápidas, en muchas ocasiones sobre cauces secos, con grandes consecuencias geomorfológicas y alta peligrosidad. El grado de riesgo viene definido por la vulnerabilidad de actividades humanas que pueden haberse instalado tanto en los propios cauces como en los conos generados en la 
desembocadura. Estas morfologías evidencian la peligrosidad activa del sistema fluvial y por ello se diagnosticaron los núcleos de población en Aragón que se encuentran sobre conos aluviales. Estos eventos de crecida son fundamentalmente estivales, también primaverales y otoñales.

b) Procesos en cursos medios y bajos del Ebro y en sus afluentes y subafluentes principales, en los que las crecidas son más largas y lentas, con hidrogramas más tendidos y complejos, pudiendo provocar inundaciones más o menos prolongadas en los llanos aluviales. El desbordamiento en amplias superficies supone una auto-laminación que atenúa el caudal punta aguas abajo. En este caso, los sectores de máximo riesgo son los que cuentan con llanos de inundación extensos, como el Ebro de meandros libres en Aragón (de Novillas a La Zaida). Son inundaciones generalmente invernales aunque en las subcuencas más orientales como las de los ríos Cinca (García Ruiz et al., 2001) ó Guadalope el mayor número de crecidas se registran en los periodos equinocciales, otoño y primavera.

c) Habría además un amplio grupo de casos de transición entre los dos tipos precedentes, correspondiendo a cursos de agua de tamaño medio, en muchas ocasiones cursos medios de ríos pirenaicos e ibéricos, cuyos procesos de crecida suelen ser considerablemente rápidos y además provocan inundaciones en fondos de valle, generando consecuencias importantes. Se pueden citar los que han sufrido procesos extremos en los últimos tiempos: Ara (Ibisate et al., 2000), Arba, Jalón, Huerva, Matarraña (Del Valle, 2002), Martín (Sánchez Fabre y Ollero, 2007), Alfambra o Guadalaviar (Sánchez Fabre et al., 2008). La estacionalidad de estos procesos es mucho más variada.

Las crecidas fluviales son procesos naturales de respuesta hidromorfológica del sistema ante una determinada situación (un evento de precipitaciones intensas, fusión nival, etc.) El proceso se convierte en riesgo cuando el hombre interviene en dicho sistema y ocupa el territorio del río, quedando expuesto a la dinámica fluvial, constriñendo su cauce a su paso por los núcleos de población, cultivando su llanura de inundación, etc. (Ollero, 2007).

La identificación de núcleos urbanos con riesgo de sufrir inundaciones se evidencia muchas veces tras un episodio de crecida, en donde se materializa dicho riesgo y es entonces cuando se toma conciencia al respecto. En otros casos la baja periodicidad de estos episodios extremos no permite este diagnóstico y, por ello, muchos núcleos de población en Aragón se encuentran en una situación de riesgo latente sin que sus habitantes tengan el recuerdo de un evento reciente (memoria histórica). Además, si un evento relevante de crecida no se ha materializado desde hace mucho tiempo (50 años o más), el comportamiento hidromorfológico del río ante este tipo de fenómeno puede ser muy diferente al del último episodio, pues la cuenca y el cauce han podido sufrir cambios en su estado que repercutan en el funcionamiento hidrológico del curso fluvial. 
El desconocimiento o menosprecio de este riesgo en muchos casos lleva a actuaciones poco sostenibles con el río y su cauce. Por ello es necesario realizar un catálogo de núcleos de población que se encuentran en situación de riesgo, que sirva como diagnóstico en la ordenación del territorio fluvial a escala autonómica.

\section{Metodología}

El diagnóstico de los núcleos de población con riesgo de inundación fluvial requiere una metodología específica que no sólo permita establecer el catálogo de aquellos núcleos objeto de este riesgo, sino evaluar la intensidad con que a priori cada uno de ellos puede verse afectado por los procesos de inundación.

La metodología ha consistido fundamentalmente en el reconocimiento de los núcleos de población con riesgo de inundación mediante fotografía aérea (fotointerpretación). Para ello se han consultado servidores web (http://sitar.aragon.es) que permiten la visualizaron de ortofotos y, uno por uno, se han analizado todos los núcleos de población de Aragón, comprobando si algún curso fluvial atraviesa o discurre cercano al núcleo urbano. Igualmente se ha consultado en el Mapa Topográfico Nacional de España (escala 1:25.000) la cota del núcleo de población y su proximidad al curso fluvial en cuestión. En los casos en los que el diagnóstico no era del todo evidente se ha recurrido a la búsqueda documental de eventos pasados de crecidas que hubiesen generado inundaciones.

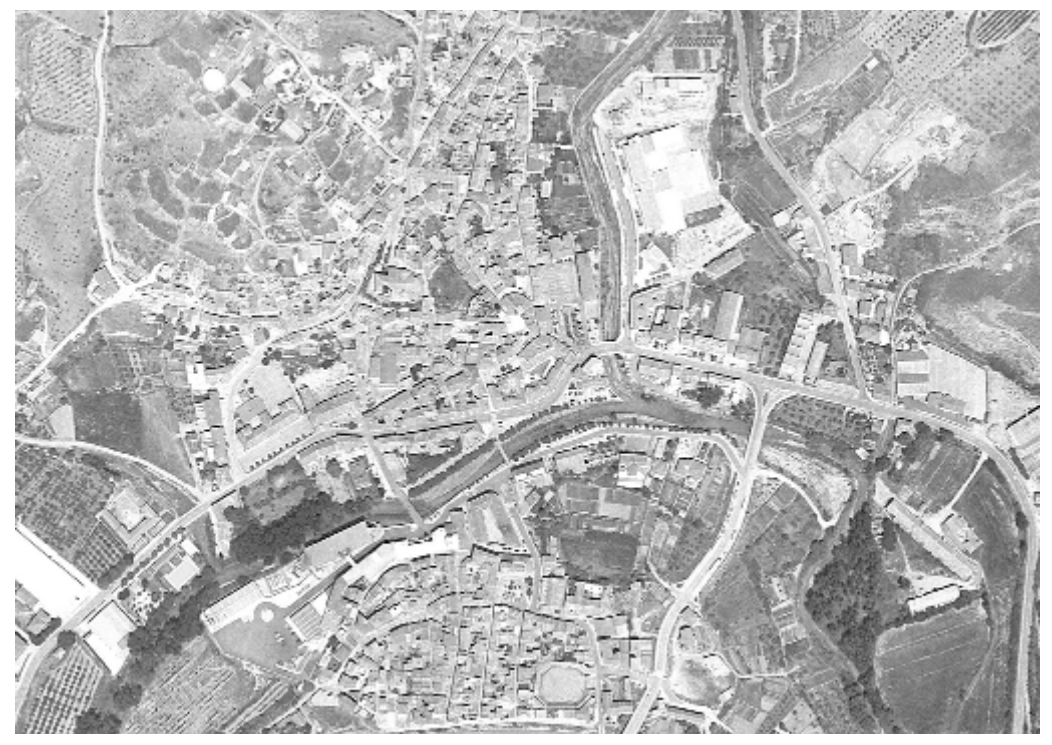

Figura 1. Confluencia del río Jalón y el Manubles en Ateca (Zaragoza). Foto: Sistema de Información Territorial de Aragón (SITAR). Gobierno de Aragón. 
Se han utilizado también otras fuentes de información para el diagnóstico de los núcleos en riesgo. Así, se ha recurrido a la consulta de bases históricas de referencia hidrológica reflejada en la publicación de la Comisión Nacional de Protección Civil para la cuenca del Ebro (1985) y para la del Júcar (1983). A través de ellos, se puede conocer la frecuencia con la que se produjeron fenómenos de inundación, es decir, se evidencia así la materialización de los riesgos potenciales en determinados espacios urbanos.

Otra variable que se ha considerado en el diagnóstico es la acción de la Administración en el mantenimiento de los cauces que presentan riesgos potenciales de desbordamiento. Así, se han recopilado todas las actuaciones que las Confederaciones Hidrográficas del Ebro y del Júcar realizan o han realizado sobre los cauces, de cara a su limpieza, reconstrucción de defensas, etc., con el objetivo de reducir su peligrosidad en crecida. La consulta para las actuaciones en la cuenca del Ebro se realizó en la página web de la Confederación a día 10/09/2007, mientras que para la del Júcar se realizó hasta el 12/09/2007. En lo que respecta a la Confederación Hidrográfica del Tajo, no se dispone de información para el territorio aragonés.

En algunos casos, las actuaciones se producen a posteriori de una crecida y consisten en la reconstrucción de defensas, retirada de escombros, aumento de la capacidad de desagüe... En otros, las actuaciones son preventivas, tratándose básicamente de limpiezas de vegetación puntuales (árboles muertos en el cauce) o continuas (de vegetación colonizadora) con el fin de evitar obstrucciones en puentes $\mathrm{u}$ otras infraestructuras. Estas actuaciones son, pese a su impacto ambiental, bien valoradas por la población local en general, siendo los propios ayuntamientos los que las demandan la mayoría de las veces.

Finalmente se trabajó con el Plan Especial de Protección Civil ante Inundaciones en la Comunidad Autónoma de Aragón aprobado en 2005 (PEPCIA a partir de aquí), que cuenta con estudios de inundabilidad e inventario de puntos de riesgo. Se incluyen así en el diagnóstico de los núcleos de población en riesgo a aquellos que el PEPCIA identificó, bien por encuesta municipal o por estudios hidráulicos. Sin embargo, este documento no analiza el riesgo de todos los cauces fluviales de Aragón, pues tan sólo se incluyen en los estudios hidráulicos de inundabilidad los tramos de los cursos regulados por un embalse aguas abajo de éste. Las encuestas que se enviaron a cada uno de los municipios aragoneses tuvieron un porcentaje de respuesta diferente por provincias, pudiendo así detectar otros núcleos en riesgo que no lo estaban por el método hidrológico-hidráulico.

\section{Diagnóstico de los núcleos de población en riesgo}

La distribución de los núcleos con riesgo de inundación se analiza desde tres perspectivas diferentes: espacial, por tipología fluvial y según la densidad de edificaciones. 


\subsection{Distribución espacial}

De los 1.729 núcleos de población que hay en Aragón, 371 se han catalogado con riesgo de inundación fluvial, lo que representa el 21,5\% del total, es decir, que aproximadamente una de cada cinco poblaciones se encuentra en una situación de riesgo hidrológico constatada (véase tabla 1 en anexo). Sin embargo, el hecho es más complejo, pues hay núcleos con más de un curso fluvial que puede provocar inundaciones, y en estos casos dicho riesgo es por tanto mayor al aumentar la peligrosidad. Así, se han catalogado hasta 449 tramos fluviales distintos que generan inundaciones. La confluencia de dos ríos en el municipio, como en Aínsa (Huesca), la existencia de dos ramblas o barrancos, como en Utrillas (Teruel) o la combinación de un río y un barranco como ocurre en Calatayud (Zaragoza), son los casos más comunes. Un 82\% de los 371 núcleos inventariados poseen riesgo hidrológico originado por un único curso fluvial. Un 15\% lo es por dos cursos y sólo el 3\% (once núcleos) lo son por tres, como Cariñena (Zaragoza) con la confluencia de tres barrancos. La gestión del riesgo y del espacio fluvial en estos casos se evidencia mucho más dificultosa.

El análisis de los núcleos en riesgo por provincias refleja que el $41 \%$ se encuentran en la de Zaragoza, un 32\% en la de Huesca y un $27 \%$ en la de Teruel. En números absolutos, en la provincia de Zaragoza no llega a haber un núcleo de población en

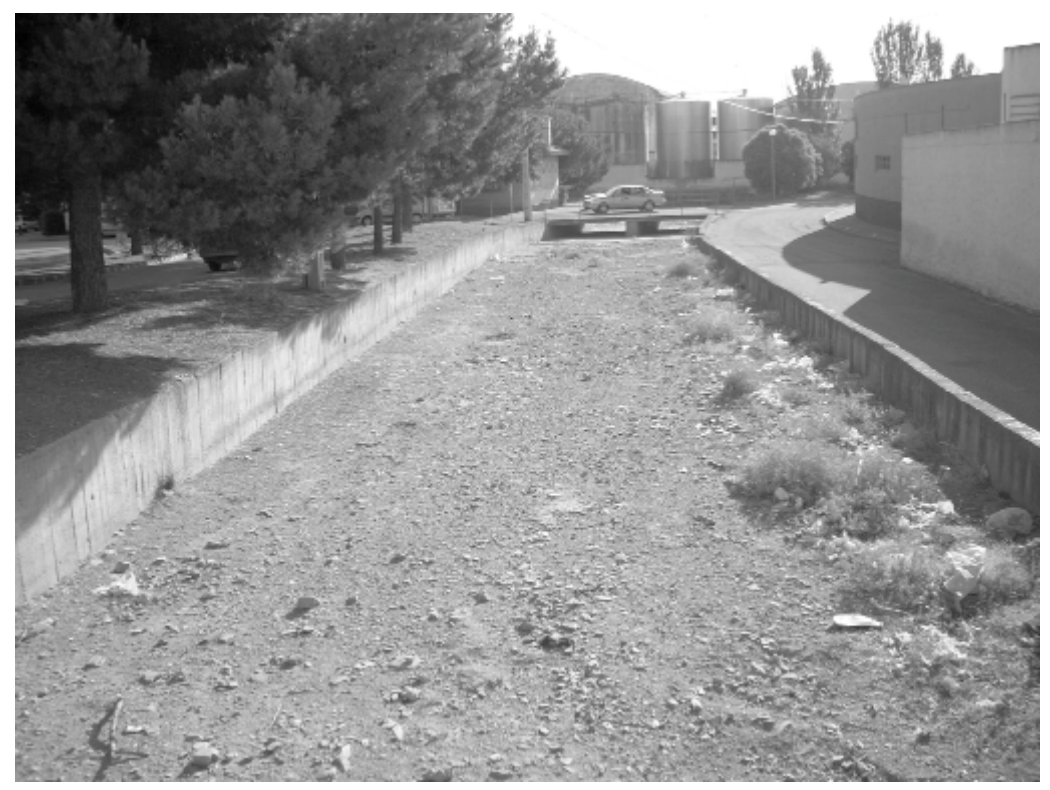

Figura 2. Barranco Cuatro Esquinas (Cariñena, Zaragoza). Foto: S. Doménech. 
riesgo de inundación fluvial por cada $100 \mathrm{~km}^{2}(0,88)$. En Huesca se reduce esta proporción $(0,75)$ y en Teruel la relación aun es menor, habiendo 0,69 núcleos por cada $100 \mathrm{~km}^{2}$. El principal factor del elevado número de casos en la provincia de Zaragoza se encuentra en la mayor superficie de las llanuras de inundación de algunos cursos fluviales grandes, como es el caso del Ebro. En zonas montañosas el poblamiento junto a los cauces fluviales es menos frecuente. Los somontanos pirenaico e ibérico son también zonas muy aptas para el cultivo, por lo que aquí los asentamientos no aparecen tan condicionados por el relieve. La provincia de Teruel se caracteriza por tener una elevada altitud media (más de 800 metros) y una configuración maciza que condiciona la red hidrográfica y los asentamientos; por ello no existen grandes ríos de llanura y los núcleos se disponen diseminados en el territorio allí donde tradicionalmente ha habido un mayor aprovechamiento agrosilvo-pastoril. También hay que señalar que muchas poblaciones no se encontraban en zonas inundables, pero han desarrollado barrios nuevos junto a los ríos, incrementando la exposición al riesgo.

En cualquier caso, resulta llamativa la equitativa distribución de casos por el territorio aragonés, que confirma que se trata de un riesgo muy extendido, registrado en todos los sistemas fluviales.

\subsection{Distribución en función de la tipología fluvial}

El territorio aragonés posee una enorme diversidad de cursos fluviales. En el inventario de núcleos en riesgo se han clasificado los casos en función del tipo de curso fluvial que atraviesa el núcleo de población. Para ello se ha empleado, como referencia inicial, la clasificación de cursos fluviales de Díaz Bea y Ollero (2005) para la Cuenca del Ebro, basada en tres variables geomorfológicas: la pendiente, la geomorfología del valle y la del cauce (estilo fluvial). Ni la pendiente ni la morfología del cauce se han considerado en el presente diagnóstico inicial, ya que no se juzga esencial a esta escala de trabajo el tipo de cauce de un río en el desbordamiento del mismo. Importa más la geomorfología del valle, y por tanto del llano de inundación, espacio potencialmente inundable en episodios de crecidas.

Así, se han diferenciado cinco tipos de cursos fluviales atendiendo a la geomorfología del valle: cursos con valle abierto extenso (A), con valle abierto de fondo encajado o semiencajado (E), con valle encajado de fondo cóncavo (V), con valle encajado de fondo plano (U) y con valle cerrado en cañón (C). Su descripción se ha realizado de forma cualitativa, sin introducir en la clasificación valores cuantitativos sobre algunos de sus elementos como la pendiente de sus vertientes o la anchura del fondo de valle (Díaz Bea y Ollero, 2005). A esta clasificación de los cursos fluviales con caudal circulante de forma habitual se debe añadir una categoría fluvial más: la de los cursos fluviales con caudal intermitente (barrancos y ramblas, B), por ser sus crecidas especialmente virulentas y peligrosas en los núcleos de población que atraviesan. 

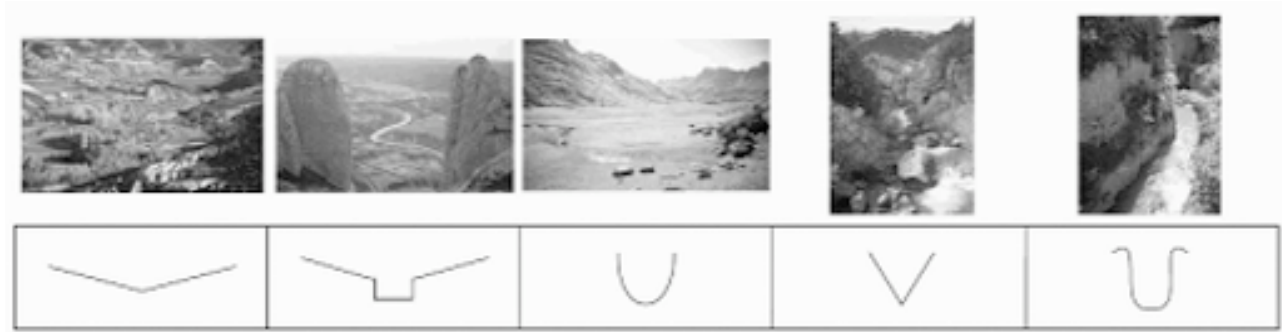

(A) $\quad(E)$

(U)

(V)

(C)

Figura 3. Tipos de cursos fluviales atendiendo a la geomorfología del valle.

De los 449 tramos fluviales peligrosos para los núcleos de población, casi el 39\% corresponde a ramblas o pequeños barrancos de caudal intermitente a lo largo del año. Es decir, cursos fluviales que experimentan crecidas relámpago, rápidas en el tiempo con un comportamiento típico flash-flood. En muchas ocasiones la baja periodicidad de estos eventos hace que no se tenga muy en cuenta al barranco, y se realizan actuaciones poco adecuadas en el espacio de inundación del mismo, lo que hace aumentar la exposición ante futuros episodios de crecidas, y por tanto aumentar el riesgo de esa población.

Este alto porcentaje de casos (más de un tercio del total de cursos fluviales inventariados) ligados a ramblas y barrancos tiene mucho que ver con la mediterraneidad climática de Aragón. Los episodios convectivos estivales son en muchas ocasiones responsables de aumentos súbitos del caudal de estos cursos,

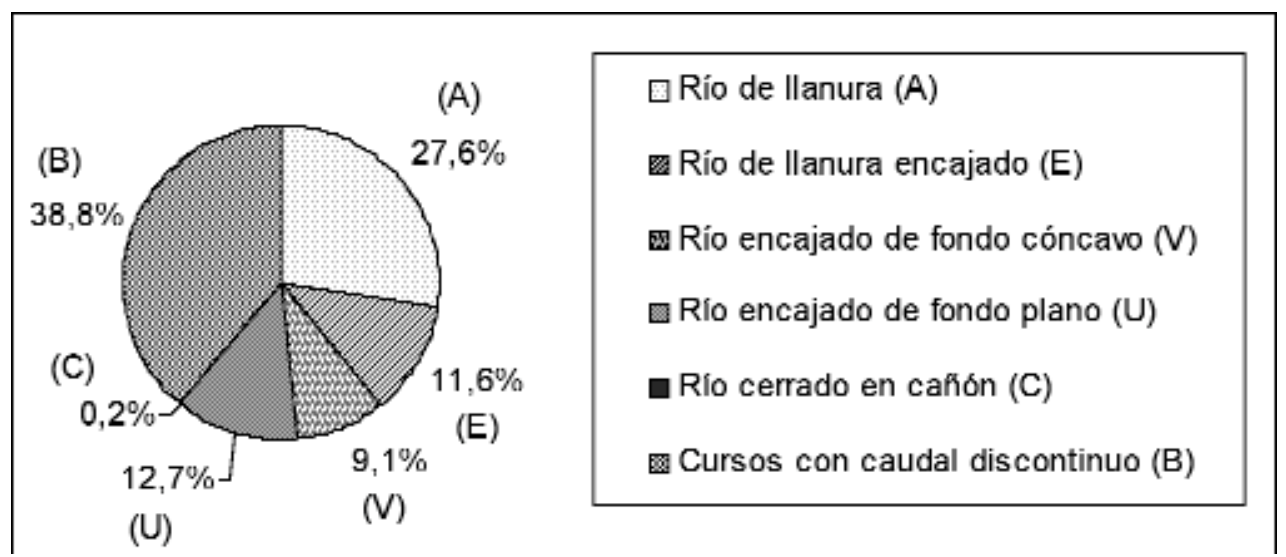

Figura 4. Distribución según la geomorfología del valle de los cursos fluviales que generan riesgos de inundación en núcleos de población de Aragón. 
debido a las intensas precipitaciones que se pueden llegar a producir en cortos espacios de tiempo (horas). Estos comportamientos torrenciales se concentran más en la Ibérica turolense pero se dan en todo el territorio aragonés, tal y como corresponde a los ámbitos mediterráneos.

Los ríos de llanura o cursos abiertos que se han diagnosticado representan el $27,6 \%$ del total de casos con riesgo de desbordamiento en poblaciones. Se trata de cursos fluviales de cauce amplio que divagan sobre una llanura de inundación extensa. El Ebro es el máximo representante, pero también caben aquí otros como los tramos bajos del Cinca, del Gállego, del Jalón, etc. El riesgo al que estos municipios se exponen procede en muchos casos del crecimiento de las ciudades en los últimos años, ocupando la llanura de inundación de estos ríos, como ocurre en el caso de Fraga en el Cinca o de Zaragoza en el Ebro.

Los ríos encajados de fondo plano representan casi el 13\% de los identificados como peligrosos para las poblaciones. Se caracterizan por un cauce que puede divagar lateralmente pero que está limitado por las paredes escarpadas del valle con pendientes abruptas que a veces tienen su origen en el modelado glaciar (Díaz Bea y Ollero, 2005). La morfología del valle en estos casos limita muchas veces la expansión urbanística del núcleo teniendo éste que ocupar las zonas más próximas al cauce, aumentando así el riesgo.

Casi un $12 \%$ de los cursos fluviales que se han catalogado como peligrosos para las poblaciones de sus riberas son ríos de llanura encajados, es decir, ríos que discurren salvando pendientes laxas pero con un fondo encajado o semiencajado, de

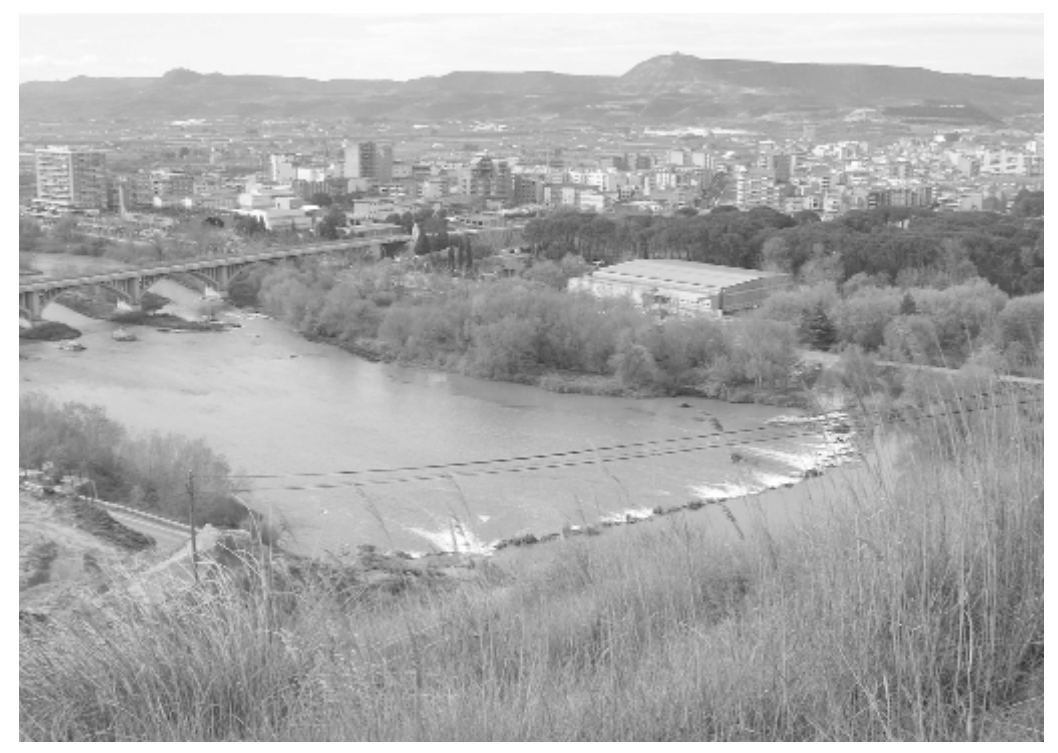

Figura 5. Zona inundable por un río de llanura: el Cinca en Fraga (Huesca). Foto: A. Ollero. 
forma que el cauce ya no puede divagar lateralmente al máximo. Suelen encajarse en las terrazas fluviales y glacis más recientes, por lo que su llanura de inundación es más reducida que en los cursos de llanura abiertos (Díaz Bea y Ollero, 2005) y el espacio potencialmente inundable de estos cursos queda limitado por la propia morfología del valle.

Los cursos de las dos categorías fluviales anteriores (casi un 25\% del total) poseen un cauce no muy amplio y suelen discurrir salvando pendientes suaves por topografías laxas con gran dedicación agropecuaria, es decir, zonas tradicionalmente humanizadas y por tanto con asentamientos cercanos al cauce. Es por ello que, conjuntamente, son las terceras tipologías fluviales más comunes en el territorio aragonés, apareciendo fundamentalmente en el somontano pirenaico y en la Ibérica, tras los barrancos de caudal intermitente y los ríos de valles extenso abiertos.

Los ríos encajados de fondo cóncavo representan el $9 \%$ de los cursos inventariados como peligrosos. Se trata de cursos fluviales que suelen salvar importantes pendientes y que están encajados en un fondo de valle con poca llanura de inundación. Son propios de áreas de montaña, característicos de cabeceras y gargantas (Díaz Bea y Ollero, 2005). La peligrosidad del sistema es elevada por lo que en la mayoría de las ocasiones se recurre a encauzar el río a su paso por el municipio para evitar desbordamientos.

Las situaciones de riesgo hidrológico en cursos fluviales con valle cerrado en cañón es muy difícil de encontrar, pues las poblaciones no se asientan en cañones

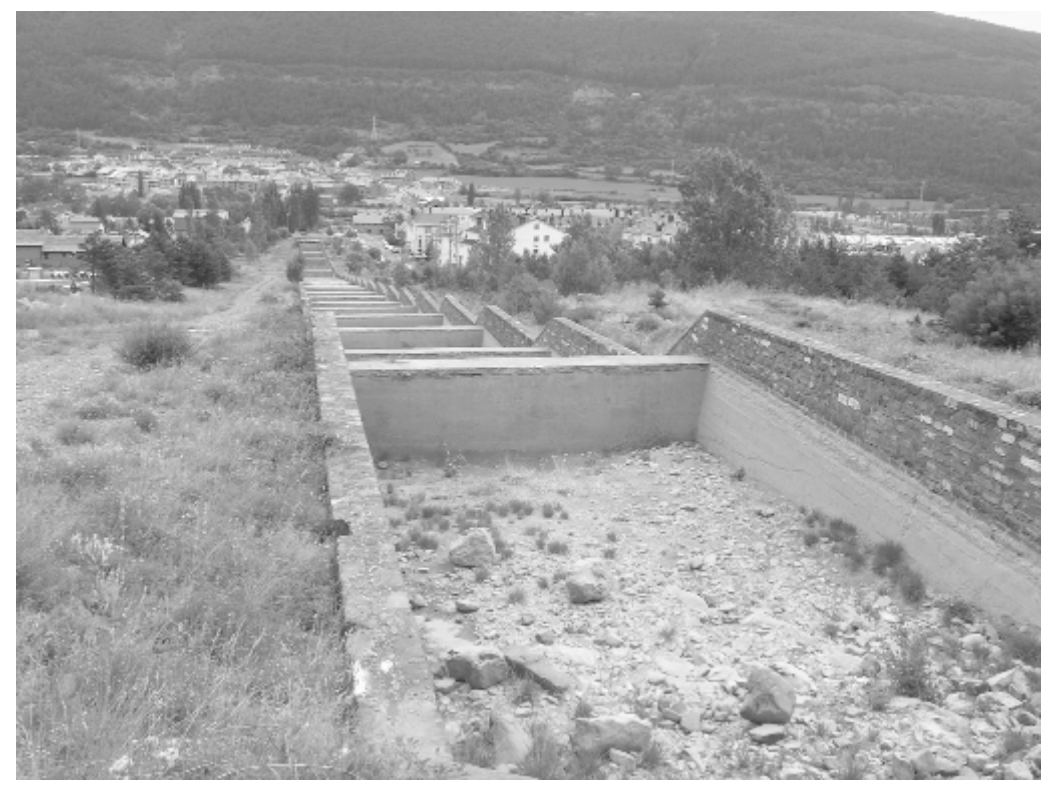

Figura 6. Cono de deyección del barranco Lierde (Villanúa, Huesca). Foto: A. Ollero. 
fluviales con poco espacio útil edificable. En Aragón se ha encontrado un solo caso que reúne estas características: el núcleo de Calmarza en la provincia de Zaragoza, en el valle del río Mesa.

En la clasificación de los riesgos según la tipología del curso fluvial hay que hacer una mención específica a una morfología fluvial indicadora del funcionamiento hidrológico: los conos aluviales. Se trata de una forma de relieve con planta en abanico que se genera allí donde el río pierde pendiente y, por un cambio acusado de la misma, deposita acarreos en crecidas. Se ligan por tanto a fenómenos hidrológicos de gran dinámica geomorfológica, como corresponden a torrentes de alta montaña y ramblas mediterráneas. La materialización de este riesgo tuvo un claro exponente en la tragedia del camping de Biescas, en el barranco de Arás (Cancer, 1996; García Ruiz et al., 1996; García Ruiz et al., 2004; Benito et al., 1998; Gutiérrez et al., 1998; Ayala-Carcedo, 2002). En este trabajo se han detectado 20 núcleos urbanos que se asientan sobre este tipo de morfología (un 5,4\% respecto del total), susceptibles de ser inundables, de los cuales un 60\% son sobre conos ligados a barrancos y ramblas, un $15 \%$ a torrentes de alta montaña, otro $15 \%$ a cursos sobre valles de fondo plano y el 10\% restante sobre conos no funcionales en ríos extensos abiertos.

\subsection{Distribución en función de la densidad de edificaciones}

Hasta ahora se han identificado los distintos tipos de cursos fluviales que potencialmente pueden ser peligrosos para los núcleos catalogados en riesgo. El diagnóstico de la población expuesta a dichos riesgos debe hacerse también de manera inicial y para ello, se ha recurrido al estudio de la densidad de poblamiento de dichos núcleos en la llanura de inundación.

El análisis de la población expuesta al riesgo mediante el cómputo total de habitantes que viven en el municipio no es factible de cara a una estimación realista del riesgo, pues no todos los habitantes del municipio viven en zonas inundables. Utilizando esta mecánica para Aragón en el año 2006, se obtiene un análisis engañoso de la realidad (véase figura 6), ya que se magnifica el riesgo de la capital aragonesa, Zaragoza, al concentrar ésta más de la mitad de la población y tampoco encontrarse sus 650.000 habitantes en zona inundable.

Así, para valorar de manera realista la población expuesta al riesgo de inundación en Aragón, no se consideró el número total de habitantes del núcleo, sino el tipo de asentamiento o densidad de edificaciones que pueden verse afectadas ante una crecida. La exposición de los bienes a las inundaciones refleja la fragilidad de la sociedad a estos procesos extraordinarios y, por tanto, hace aumentar el riesgo.

Como ya se ha dicho, se identificaron 449 tramos fluviales que pueden generar inundaciones en 371 núcleos de población. Un 43\% de estos cursos afectarían a un poblamiento disperso con una densidad baja de edificaciones (DB). Bajo esta denominación se incluyeron las viviendas aisladas que pueden ser inundadas o 


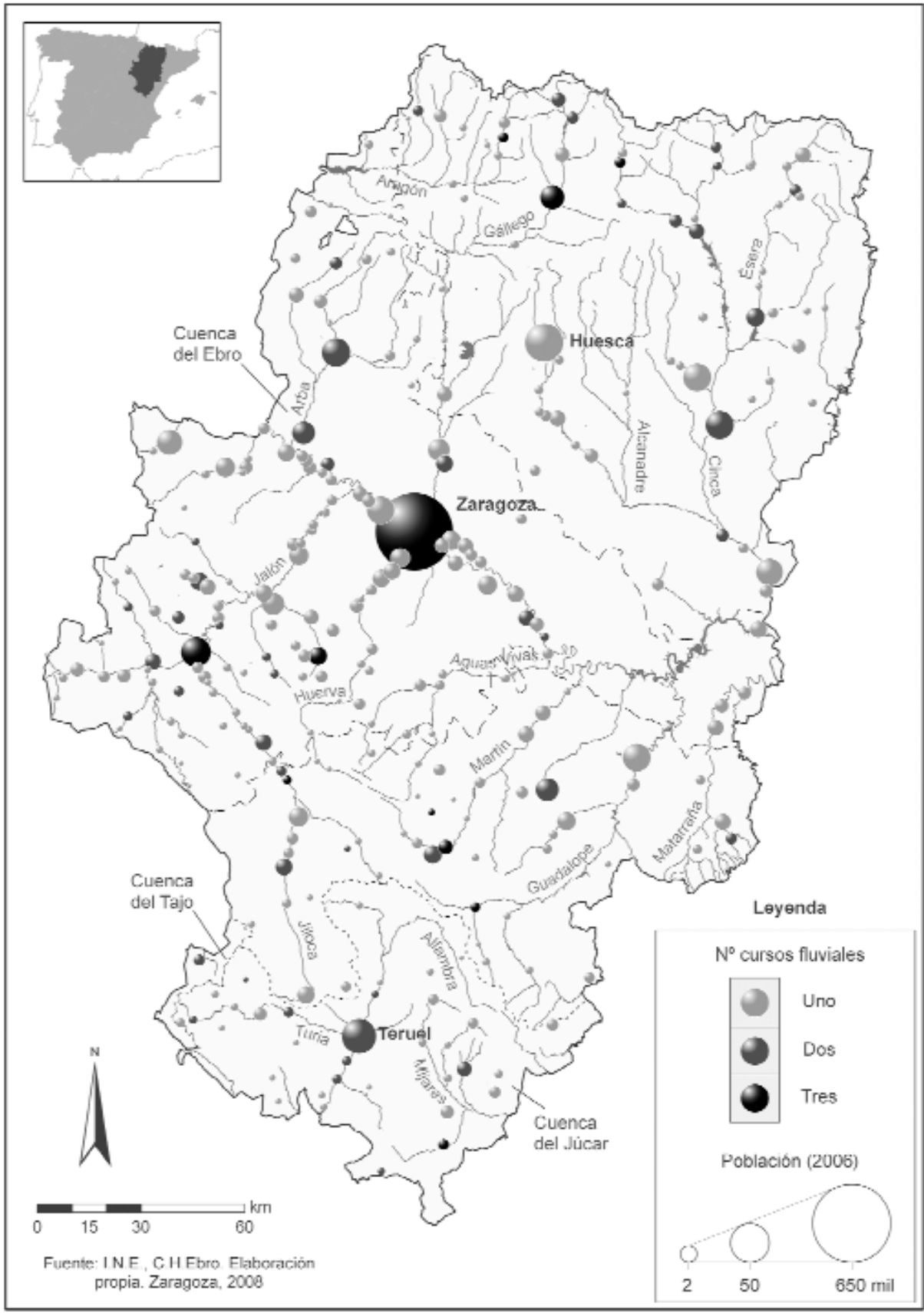

Figura 7. Población expuesta al riesgo de inundación fluvial en Aragón a escala municipal. 
edificaciones que no forman un núcleo compacto. Destaca el amplio porcentaje de casos que quedan bajo esta denominación porque la mayor parte de los núcleos rurales del territorio aragonés poseen un poblamiento diseminado. También se incluyeron en este grupo urbanizaciones ilegales que suelen corresponder a segundas residencias o pequeñas fincas de recreo.

Se consideró como densidad media de poblamiento (DM) a las urbanizaciones consolidadas pero que se encontraban separadas del núcleo principal, las zonas deportivas, industriales o núcleos rurales consolidados. Así, el 38\% de las inundaciones fluviales afectaría a asentamientos de estas características.

Por último, las inundaciones que se pueden dar en núcleos urbanos consolidados representan el 19\% del total de casos. Así, se consignó como densidad de edificaciones alta (DA) a todos los núcleos de población con una trama urbana densa y compacta. Existen pocas ciudades como tales en Aragón y por ello se categorizaron pocos ejemplos de este tipo de poblamiento en núcleos con riesgo hidrológico. Sin embargo en estas poblaciones, la vulnerabilidad de los edificios es muchas veces mayor, al disponer éstos de sótanos y garajes que se inundan con frecuencia.

\section{Evaluación de los núcleos de población en riesgo}

Una vez se han identificado y diagnosticado los núcleos de población en riesgo de sufrir una inundación fluvial en Aragón, es preciso valorar dicho riesgo para jerarquizar los núcleos identificados y actuar frente al riesgo en consecuencia. El análisis de los núcleos en riesgo según su distribución espacial, el tipo de curso fluvial y la densidad de poblamiento debe completarse con otras variables que permitan validar el riesgo inicialmente detectado.

\section{1. Índice sintético de riesgo}

Tal y como se ha apuntado, se tuvieron en cuenta diversas variables en la evaluación de los núcleos de población en riesgo por inundaciones fluviales, tales como las referencias hidrológicas de avenidas históricas o su inclusión en estudios como el PEPCIA. Así se intentó jerarquizar la importancia del riesgo en los núcleos en donde ya se habían materializado crecidas históricas o poseían actuaciones en el cauce encaminadas a la minimización de los riesgos.

Con estas variables se ha elaborado un índice sintético que ponderase los núcleos según su situación de riesgo. Si un núcleo de población sólo se había detectado en riesgo mediante la fotointerpretación anterior, se le asignó un valor de riesgo bajo. Si además dicho núcleo poseía registro de crecidas históricas en la obra de la Comisión Nacional de Protección Civil, se le consignó un valor de riesgo medio. Si también registraba actuaciones en el cauce llevadas acabo por la Confederación Hidrográfica o estaba incluido en riesgo en el PEPCIA, se le asignó un valor de riesgo alto. 
Finalmente, si el núcleo contaba con actuaciones en el cauce además de su inclusión en el PEPCIA, junto con las demás variables, se adjudicaba un valor muy alto de riesgo.

Aunque habría sido importante definir el nivel de riesgo contando también con información de la frecuencia de la producción de las crecidas en cada núcleo (periodicidad), resulta muy complejo ya que sólo en algunos existen estaciones de aforo próximas. También se puede recurrir a la prensa diaria para analizar esta frecuencia. Se prevé incluir este parámetro en el proceso de desarrollo de la presente línea de investigación. En el presente análisis se ha intentado estimar dicha frecuencia en la evaluación final del riesgo al considerar el número de inundaciones pasadas, constatadas en los documentos manejados (Comisión Nacional de Protección Civil, 1983, 1985).

La figura 7 ilustra la distribución de los núcleos de población en riesgo según el valor de este índice sintético. Se puede observar cómo ahora la distribución del riesgo de inundación fluvial no aparece sesgada por el peso demográfico de ninguna entidad y se contempla la existencia de dos grandes ejes fluviales con riesgo significativos para las poblaciones ribereñas. Se trata del eje del Ebro en una primera instancia y el del Jalón-Jiloca, en otro orden de importancia, al encontrar en este segundo corredor menos población expuesta al riesgo. En el resto del territorio no se contemplan rasgos definitorios de la distribución del riesgo por inundación asociado a otros cursos fluviales, y tan sólo en las cabeceras de los afluentes pirenaicos del Ebro se dan mayores situaciones de riesgo. En el resto de cuencas las situaciones de riesgo son menores y se encuentran más dispersas.

\subsection{Evaluación final del riesgo}

El índice anteriormente descrito permite jerarquizar los núcleos de acuerdo a una serie de variables que no se habían considerado en la identificación de los núcleos en riesgo. Por ello, a continuación se presenta una evaluación final de este riesgo considerando la variable "densidad de poblamiento" del análisis inicial, así como las que integra el índice sintético (avenidas históricas, actuaciones en el cauce y su inclusión en el PEPCIA). Así se consigue tener una visión de conjunto de los núcleos en riesgo de sufrir inundaciones fluviales en Aragón.

La forma de evaluar el riesgo global de los núcleos ha consistido en reclasificar las variables que antes se han mencionado.

- La densidad de poblamiento que puede verse afectada en una inundación, expresiva de la mayor o menor población expuesta, se ha reclasificado de la siguiente forma: se asignaron tres puntos a los casos con densidad alta, dos a los de media y un punto a los de baja.

- El número de avenidas históricas (entendiendo como tales las catalogadas en los inventarios realizados por la Comisión Nacional de Protección Civil en 1983 y 1985) representativo de la peligrosidad del curso fluvial, se reclasificó asignando 


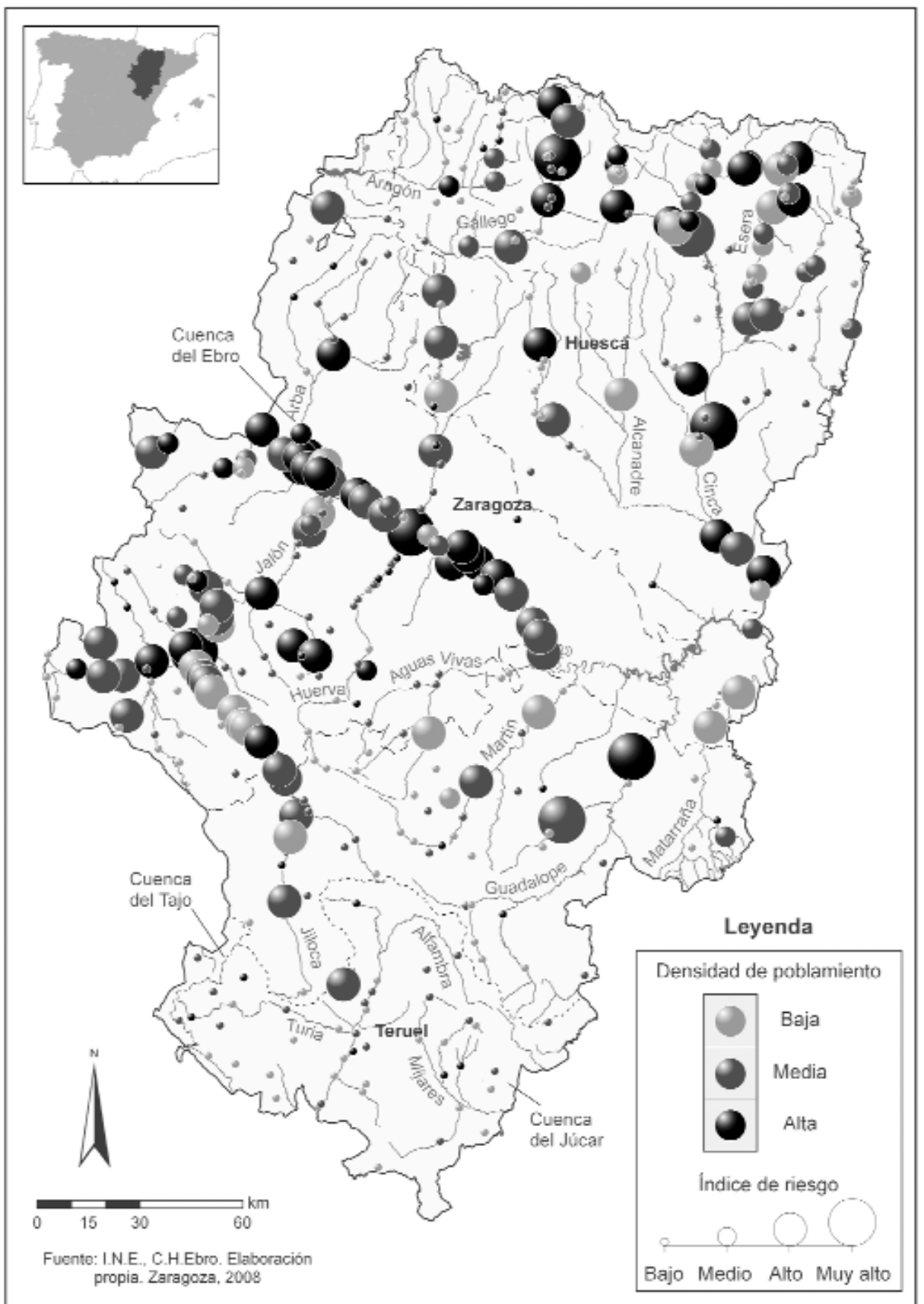

Figura 8. Evaluación del riesgo de inundación fluvial en núcleos de población de Aragón. 
un punto si el municipio poseía entre una y cinco avenidas, dos puntos si tenía entre seis y diez, y tres puntos cuando se daban once casos o más.

- Se añadió el valor del índice sintético de riesgo anteriormente consignado reclasificándolo de la siguiente forma: valor de riesgo bajo, un punto; riesgo medio, dos puntos; riesgo alto, tres puntos, y valor de riesgo muy alto, cuatro puntos.

Finalmente se sumaron los puntos consignados en cada una de las tres variables y se obtuvo un valor cuantitativo de riesgo global para cada núcleo de población.

Cabe pensar que se potenció demasiado el peso de las avenidas históricas en la jerarquización de los núcleos en riesgo, al considerarse tanto en el índice sintético como el número total de las mismas, pero ello es así porque la peligrosidad del sistema fluvial queda así expresada de mejor forma que con el número de cursos fluviales que atraviesa el núcleo. Así por un lado, se valora que el núcleo haya sido inundado ya alguna vez, con su inclusión en el índice sintético, y por otro lado, se pondera la frecuencia de dichas inundaciones con el número total de avenidas históricas registradas, considerando que una frecuencia de más de diez episodios supone el máximo riesgo.

Todas estas variables permitieron evaluar el riesgo de los núcleos de población con un valor global de riesgo que oscila entre dos y diez puntos. No existe el valor nulo de riesgo, pues éste estaría asignado para los núcleos que no están en riesgo, es decir, los que no se han identificado mediante fotointerpretación y por tanto no son objeto de estudio. El valor de riesgo "uno" tampoco se ha consignado porque cualquier núcleo de población que se ha detectado en riesgo, tiene como mínimo un punto en el índice sintético y otro en la densidad de poblamiento.

Tabla 2. Núcleos de población con mayor riesgo.

\begin{tabular}{|l|c|l|c|}
\hline $\begin{array}{l}\text { Entidad } \\
\text { de población }\end{array}$ & Provincia & Curso fluvial & Riesgo final \\
\hline Calatayud & Z & Jalón, Barranco las Pozas, Jiloca & 10 \\
Alcañiz & T & Guadalope & 10 \\
Zaragoza & Z & Ebro, Huerva, Gállego & 10 \\
Ateca & Z & Manubles, Jalón & 9 \\
Daroca & Z & Arroyo de la Mina, Jiloca & 9 \\
Biescas & $\mathrm{H}$ & Gállego & 9 \\
Barbastro & $\mathrm{H}$ & Vero & 9 \\
Aínsa & $\mathrm{H}$ & Ara, Cinca & 9 \\
Novillas & $\mathrm{Z}$ & Ebro & 8 \\
Boquiñeni & $\mathrm{Z}$ & Ebro & 8 \\
Ejea de los Caballeros & $\mathrm{Z}$ & Arba de Luesia, Arba de Biel & 8 \\
Monzón & $\mathrm{H}$ & Cinca, Sosa & 8 \\
Boltaña & $\mathrm{H}$ & Barranco de San Martín, Ara & 8 \\
Fraga & $\mathrm{H}$ & Cinca & \\
\hline
\end{tabular}


Tal y como se puede comprobar, los núcleos con mayor riesgo global son aquellos que a lo largo del tiempo han sufrido con mayor recurrencia las crecidas de los cursos fluviales que los atraviesan. Las crecidas históricas registradas en grandes sistemas fluviales como el Ebro o Cinca están mejor documentadas que las que generan los pequeños barrancos y ramblas, mucho más rápidas en el tiempo. Por ello los núcleos ribereños de estos grandes sistemas poseen globalmente un valor final de riesgo mayor. La consideración en esta evaluación final del riesgo de otras variables como la densidad de poblamiento actual, que puede verse afectada por una crecida, así como el índice sintético de riesgo elaborado, permite evaluar la existencia de un riesgo presente y no sólo la evidencia de una situación pasada de riesgo.

El procedimiento de evaluación realizado, sencillo y cualitativo, puede ser útil fundamentalmente para seleccionar casos de estudio en los que profundizar posteriormente el análisis y proponer soluciones de mitigación.

\section{Conclusiones y estrategias de futuro}

El diagnóstico y evaluación que se ha realizado de los núcleos de población con riesgo de inundación debe ser aprovechado en la ordenación del territorio aragonés al igual que se ha hecho en otras comunidades (Díez Herrero et al., 2008a). Tal y como se ha puesto de manifiesto en la presente investigación, existe un gran número de núcleos de población en riesgo de sufrir inundaciones fluviales en la Comunidad Autónoma de Aragón. Que uno de cada cinco núcleos tenga esta problemática debe hacer reflexionar acerca del comportamiento humano en los sistemas fluviales.

Los núcleos que se han diagnosticado en riesgo están amenazados, en su mayoría, por cursos de caudal intermitente, no aforados y por tanto poco predecibles en tiempo real. Estas crecidas son muy rápidas en el tiempo y por ello hay que estar preparados para poder actuar con rapidez, pues el periodo de decisión es muy corto. Así mismo, el riesgo aumenta en los núcleos por donde discurre más de un curso fluvial ya que la coincidencia de sus crecidas puede provocar daños catastróficos en las poblaciones. El prototipo de núcleo en riesgo que se ha catalogado responde a uno que se encuentra en riesgo por un solo curso fluvial de caudal intermitente (barranco o rambla) y cuyas crecidas afectan a una densidad de poblamiento baja, es decir, no hay mucha población expuesta a dichas inundaciones.

La consideración de los espacios fluviales en la ordenación del territorio a escala local no se ha tenido en cuenta muchas veces. Se ha especulado con el suelo aumentando la exposición, basándose en una falsa sensación de seguridad que se apoya en la regulación de los caudales por embalses, obras de ingeniería para el control del río y sus crecidas, y el prolongado periodo de retorno de las inundaciones en muchos cursos fluviales. Es necesario por tanto enfocar un cambio en la gestión del riesgo en estos núcleos de población. 
Todos los casos que se han inventariado con riesgo de inundaciones deberían contemplar una ordenación de este espacio fluvial a su paso por los núcleos de población. Ello no significa que se tengan que hacer canalizaciones o desvíos, sino una combinación de medidas estructurales y no estructurales, como la ordenación del llano de inundación. Bien es cierto que la gestión del riesgo no debe hacerse sólo en un punto concreto, sino con una perspectiva global de la cuenca, el cauce y sus riberas, es decir, del sistema hidrogeomorfológico, pues la peligrosidad del curso fluvial en un núcleo de población concreto se condiciona por la cuenca y no sólo por el cauce aguas arriba y al paso por dicho municipio.

El diagnóstico y evaluación de los núcleos de población en riesgo por inundaciones fluviales, plasmado en el presente trabajo, ha de servir para iniciar una línea científico-técnica en estudios hidrológicos a escala local que se integren en la ordenación del territorio municipal de cara a minimizar los riesgos naturales en dichos núcleos de población.

El desarrollo de esta investigación se completará con trabajo de campo, con el análisis de casos concretos y con la ampliación de la metodología de evaluación del riesgo por inundaciones fluviales utilizando más parámetros que los empleados en la presente (Díez Herrero et al., 2006; Díez Herrero et al., 2008b).

\section{Agradecimientos}

Esta investigación se integra en una Beca de Postgrado para la Formación de Profesorado Universitario (F.P.U.) del Ministerio de Educación y Ciencia, bajo el título "Inundaciones fluviales en espacios urbanos de Aragón: evaluación del riesgo, cartografía y modelos de gestión" (Ref: AP2006-00554).

\section{Bibliografía}

Ayala-Carcedo, F.J. (2002). La inundación torrencial catastrófica del camping "Las Nieves" del 7 de Agosto de 1996 en el cono de deyección del Arás (Biescas, Pirineo Aragonés). En Ayala-Carcedo, F.J. y Olcina, J. (coords.) Riesgos Naturales, Barcelona, Ariel, p. 889-912.

Benito, G., Grodek, T. \& Enzel, Y. (1998). The geomorphic and hydrologic impacts of the catastrofic failure of flood-control-dams during the 1996-Biescas flood (Central Pyrenees, Spain). Zeitschrift für Geomorphologie, 42 (4), 417-437.
Cancer, L. (1996). La catástrofe del barranco de Arás (7/8/1996). Procesos naturales e hipótesis explicativa. Geographicalia, 33, 51-71.

Comisión Nacional de Protección Civil (1983). Estudio de inundaciones históricas. Mapa de riesgos potenciales. Cuenca del Júcar. Dirección General de Obras Hidráulicas.

Comisión Nacional de Protección Civil (1985). Estudio de inundaciones históricas. Mapa de riesgos potenciales. Cuenca del Júcar. Dirección General de Obras Hidráulicas.

Del Valle, J. (2002). Las intensas lluvias y 
riadas del 23 al 25 de octubre de 2000 en el SE de la cuenca del Ebro. Seguimiento y gestión mediante el S.A.I.H. de la C.H.E. VII Reunión Nacional de Climatología, Albarracín, A.G.E., p. 93-98,

Del Valle, J., Ollero, A y Sánchez Fabre, M. (2007). Atlas de los ríos de Aragón. Zaragoza, Prames.

Díaz Bea, E. y Ollero, A. (2005). Metodología para la clasificación geomorfológica de los cursos fluviales de la cuenca del Ebro. Geographicalia, 47, 23-45.

Díez Herrero, A., Laín, L. y Llorente, M. (eds., 2006). Mapas de peligrosidad de avenidas $e$ inundaciones. Métodos, experiencias y aplicación. Madrid, Instituto Geológico y Minero de España.

Díez Herrero, A., Garrote, J., Baíllo, R., Laín, L., Mancebo, M.J. y Pérez Cerdán, F. (2008a). Análisis del riesgo de inundación para planes autonómicos de protección civil: RICAM. En Galindo, I., Laín, L. y Llorente, M. (eds.) El estudio y la gestión de los riesgos geológicos, Madrid, Instituto Geológico y Minero de España, p. 53-69.

Díez Herrero, A., Laín, L. y Llorente, L. (2008b). Mapas de peligrosidad por avenidas e inundaciones. Guía metodológica para su aplicación. Madrid, Instituto Geológico y Minero de España.

García Ruiz, J.M., White, S., Martí-Bono, C. Valero, B., Errea. M.P. y Gómez Villar, A. (1996). La catástrofe del barranco de Arás (Biescas, Pirineo Aragonés) y su contexto espacio-temporal. Zaragoza, Instituto Pirenaico de Ecología

García Ruiz, J.M., Beguería, S., López-Moreno, J.I., Lorente, A. y Seeger, M. (2001). Los recursos hidricos superficiales del Pirineo aragonés y su evolución reciente. Logroño, Geoforma Ediciones.

García Ruiz, J.M., White, S., Martí-Bono, C., Valero, B., Errea, M.P. y Gómez Villar, A. (2004). La avenida del Barranco de Arás y los riesgos hidrológicos en el Pirineo central español. En Peña, J.L., Longares,
L.A. y Sánchez Fabre, M. (eds.) Geografía Física de Aragón. Aspectos generales y temáticos, Zaragoza, Universidad de Zaragoza e Institución Fernando el Católico, p.131-140.

Gutiérrez, F., Gutiérrez, M. y Sancho, C. (1998). Geomorphological and sedimentological analysis of a catastrophic flash flood in the Arás drainage basin (Central Pyrenees, Spain). Geomorphology, 22, 265283.

Ibisate, A., Ollero, A. y Díaz Bea, E. (2001). Las crecidas del río Ara y el evento extraordinario de diciembre de 1997. Jornadas 2000: El río Ara es de todos, Zaragoza, Asociación Río Ara, p. 65-78.

Ollero, A. (2000). Crecidas fluviales en la cuenca del Ebro desde 1980: estado de la cuestión, principales eventos y sistemas de prevención. Serie Geográfica, 9, 151162.

Ollero, A. (2006). Inundaciones y ordenación del territorio fluvial en la cuenca del Ebro. En Chastagnaret, G. y Gil Olcina, A. (eds.) Riesgo de inundaciones en el Mediterráneo occidental, Madrid, Casa de Velásquez, p.239-272.

Ollero, A. (2007). Territorio fluvial. Diagnóstico y propuesta para la gestión ambiental $y$ de riesgos en el Ebro y los cursos bajos de sus afluentes. Bilbao, Bakeaz.

Pujadas, J. (2002). Las inundaciones en España: Impacto económico y gestión del riesgo. En Ayala-Carcedo, F.J. y Olcina, J. (coords.) Riesgos Naturales, Barcelona, Ariel, p. 879-888.

Sánchez Fabre, M., Ollero, A. y Del Valle, J. (2004) La red fluvial de Aragón. En Peña, J.L. Longares, L.A. y Sánchez Fabre, M. (eds.). Geografía Física de Aragón. Aspectos generales y temáticos. Zaragoza, Universidad de Zaragoza e Institución Fernando el Católico, p. 55-70.

Sánchez Fabre, M. y Ollero, A. (2007). Caracterización y evaluación ambiental del 
río Martín y sus afluentes. Revista de Sánchez Fabre, M., Ollero, A. y Lozano, M.V. Andorra, 7, 85-104. (2008) El río Guadalaviar: su comportamiento hidrológico. Rehalda, 7, 37-52.

Anexo

Tabla 1. Catálogo de núcleos de población con riesgo de sufrir inundaciones fluviales en Aragón.

\begin{tabular}{|c|c|c|c|c|}
\hline Entidad de población & Provincia & Curso fluvial & $N^{o}$ cursos & Densidad \\
\hline Abanto & Z & Ortiz & 1 & Baja \\
\hline Agón & Z & Huecha & 1 & Media \\
\hline Aguarón & Z & Barranco de Aguarón & 1 & Alta \\
\hline Aguilón & Z & Barranco del Erasno & 1 & Alta \\
\hline Aínsa & $\mathrm{H}$ & Ara, Cinca & 2 & Media \\
\hline Alastuey & $\mathrm{H}$ & Barranco de Alastuey & 1 & Baja \\
\hline Albalate del Arzobispo & $\mathrm{T}$ & Martín & 1 & Media \\
\hline Albarracín & $\mathrm{T}$ & Guadalaviar o Turia & 1 & Media \\
\hline Alberite de San Juan & Z & Huecha & 1 & Baja \\
\hline Alcalá de Ebro & Z & Ebro & 1 & Alta \\
\hline Alcalá de la Selva & $\mathrm{T}$ & Alcalá o Balbona & 1 & Media \\
\hline Alcañiz & $\mathrm{T}$ & Guadalope & 1 & Alta \\
\hline Alcorisa & $\mathrm{T}$ & Guadalopillo & 1 & Media \\
\hline Alcubierre & $\mathrm{H}$ & Barranco & 1 & Media \\
\hline Aldehuela de Liestos & Z & Barranco de los Regachos & 1 & Baja \\
\hline Alfajarín & Z & Barranco de la Virgen & 1 & Alta \\
\hline Alfamén & Z & Barranco de las Planas & 1 & Media \\
\hline Alfocea & Z & Ebro, Barranco de los Lecheros & 2 & Media \\
\hline Alhama de Aragón & Z & Jalón & 1 & Media \\
\hline Aliaga & $\mathrm{T}$ & $\begin{array}{l}\text { Aliaga, Guadalope, Barranco } \\
\text { de la Virgen }\end{array}$ & 3 & Baja \\
\hline Alloza & $\mathrm{T}$ & Barranco de los Cañizares & 1 & Media \\
\hline Almochuel & Z & Aguas Vivas & 1 & Baja \\
\hline Almonacid de la Cuba & $\mathrm{Z}$ & Barranco Barcalién & 1 & Media \\
\hline Almuniente & $\mathrm{H}$ & Flumen & 1 & Baja \\
\hline Alobras & $\mathrm{T}$ & Barranco del Hornillo & 1 & Baja \\
\hline Alpartir & Z & Alpartir & 1 & Media \\
\hline Andorra & $\mathrm{T}$ & $\begin{array}{l}\text { Barranco del Regallo, } \\
\text { Bco de los Molinos }\end{array}$ & 2 & Alta \\
\hline Aniñón & Z & $\begin{array}{l}\text { Barranco de Acuadrón, } \\
\text { Arroyo El Fresno }\end{array}$ & 2 & Media \\
\hline Ansó & $\mathrm{H}$ & Barranco de la Fuente, Veral & 2 & Baja \\
\hline Anzánigo & $\mathrm{H}$ & Gállego & 1 & Media \\
\hline Ara & $\mathrm{H}$ & Arroyo de Abena & 1 & Baja \\
\hline
\end{tabular}




\begin{tabular}{|c|c|c|c|c|}
\hline Entidad de población & Provincia & Curso fluvial & $N^{o}$ cursos & Densidad \\
\hline Aragüés del Puerto & $\mathrm{H}$ & Osia & 1 & Baja \\
\hline Arcos de las Salinas & $\mathrm{T}$ & Arroyo Torrijana, Barranco & 2 & Baja \\
\hline Aréns de Lledó & $\mathrm{T}$ & Algas & 1 & Baja \\
\hline Ariza & Z & Barranco de la Alayera & 1 & Alta \\
\hline Aso de Sobremonte & $\mathrm{H}$ & Arás & 1 & Baja \\
\hline Atea & Z & Barranco de Valverde & 1 & Baja \\
\hline Ateca & Z & Manubles, Jalón & 2 & Alta \\
\hline Aurín & $\mathrm{H}$ & Aurín & 1 & Media \\
\hline Azanuy & $\mathrm{H}$ & Arroyo Sosa de Azanuy & 1 & Media \\
\hline Azuara & Z & Cámaras & 1 & Media \\
\hline Badules & $Z$ & Huerva & 1 & Baja \\
\hline Báguena & $\mathrm{T}$ & Jiloca, Rambla de Anento & 2 & Media \\
\hline Ballobar & $\mathrm{H}$ & Alcanadre, Barranco de & & \\
\hline & & Valdecarreta & 2 & Alta \\
\hline Baños de Panticosa & $\mathrm{H}$ & Caldarés & 1 & Baja \\
\hline Bara & $\mathrm{H}$ & Alcanadre & 1 & Baja \\
\hline Barbastro & $\mathrm{H}$ & Vero & 1 & Alta \\
\hline Bárboles & Z & Jalón & 1 & Media \\
\hline Barués & Z & Arroyo de Castiliscar & 1 & Baja \\
\hline Beceite & $\mathrm{T}$ & Matarraña, Barranco dels Olivars & 2 & Media \\
\hline Belmonte de Gracián & Z & Perejiles & 1 & Media \\
\hline Benabarre & $\mathrm{H}$ & Guart & 1 & Media \\
\hline Benasque & $\mathrm{H}$ & Ésera & 1 & Alta \\
\hline Berge & $\mathrm{T}$ & Guadalopillo & 1 & Baja \\
\hline Besiáns & $\mathrm{H}$ & Ésera & 1 & Baja \\
\hline Betés de Sobremonte & $\mathrm{H}$ & Betés & 1 & Media \\
\hline Bezas & $\mathrm{T}$ & Barranco de las Quemadas & 1 & Baja \\
\hline Biel & $\mathrm{Z}$ & Arba de Biel & 1 & Media \\
\hline Bielsa & $\mathrm{H}$ & Barrosa, Cinca & 2 & Media \\
\hline Biescas & $\mathrm{H}$ & Gállego & 1 & Alta \\
\hline Bijuesca & $\mathrm{Z}$ & Manubles & 1 & Alta \\
\hline Biota & $\mathrm{Z}$ & Arba de Luesia & 1 & Media \\
\hline Bisaurri & $\mathrm{H}$ & Barranco & 1 & Alta \\
\hline Blesa & $\mathrm{T}$ & Aguas Vivas & 1 & Baja \\
\hline Boltaña & $\mathrm{H}$ & Barranco de San Martín, Ara & 2 & Alta \\
\hline Bono & $\mathrm{H}$ & Noguera Ribagorzana & 1 & Baja \\
\hline Boquiñeni & Z & Ebro & 1 & Alta \\
\hline Borau & $\mathrm{H}$ & Lubierre & 1 & Alta \\
\hline Borja & Z & Barranco de Arbolitas & 1 & Alta \\
\hline Botorrita & $Z$ & Huerva & 1 & Baja \\
\hline Brea de Aragón & $\mathrm{Z}$ & Aranda & 1 & Media \\
\hline Broto & $\mathrm{H}$ & $\begin{array}{l}\text { Ara, Bco. de Arán o del Furco, } \\
\text { Bco. Sorrosal }\end{array}$ & 3 & Alta \\
\hline Bueña & $\mathrm{T}$ & Barranco del Salto & 1 & Media \\
\hline Buesa & $\mathrm{H}$ & Barranco de Buesa & 1 & Baja \\
\hline
\end{tabular}




\begin{tabular}{|c|c|c|c|c|}
\hline Entidad de población & Provincia & Curso fluvial & $N^{o}$ cursos & Densidad \\
\hline Bulbuente & Z & Huecha & 1 & Media \\
\hline Burbáguena & $\mathrm{T}$ & Jiloca, Rambla del Puerto, & & \\
\hline & & Rambla de San Francisco & 3 & Media \\
\hline Cabañas de Ebro & Z & Ebro & 1 & Media \\
\hline Cabra de Mora & $\mathrm{T}$ & Balbona & 1 & Baja \\
\hline Cadrete & Z & Huerva & 1 & Alta \\
\hline Calamocha & $\mathrm{T}$ & Jiloca & 1 & Media \\
\hline Calatayud & $\mathrm{Z}$ & Jalón, Barranco las Pozas, Jiloca & 3 & Alta \\
\hline Caldearenas & $\mathrm{H}$ & Gállego & 1 & Media \\
\hline Calmarza & Z & Mesa & 1 & Baja \\
\hline Calomarde & $\mathrm{T}$ & Río de la Fuente del Berro & 1 & Media \\
\hline Campamento & $\mathrm{H}$ & Noguera Ribagorzana & 1 & Baja \\
\hline Campo & $\mathrm{H}$ & Ésera & 1 & Media \\
\hline Camporrells & $\mathrm{H}$ & Barranco del Molino de Pubil & 1 & Media \\
\hline Candanchú & $\mathrm{H}$ & Aragón & 1 & Baja \\
\hline Canfranc & $\mathrm{H}$ & Aragón & 1 & Media \\
\hline Canfranc-Estación & $\mathrm{H}$ & Aragón & 1 & Media \\
\hline Capella & $\mathrm{H}$ & Isábena & 1 & Media \\
\hline Carenas & Z & Piedra & 1 & Baja \\
\hline Cariñena & Z & $\begin{array}{l}\text { Frasno, Bco. Cuatro Esquinas, } \\
\text { Bco. Degollada }\end{array}$ & 3 & Alta \\
\hline Castejón de las Armas & $\mathrm{Z}$ & Piedra & 1 & Media \\
\hline Castejón de Sos & $\mathrm{H}$ & Ésera, Barranco de Urmella & 2 & Media \\
\hline Castel de Cabra & $\mathrm{T}$ & Río del Hurón & 1 & Baja \\
\hline Castelnou & $\mathrm{T}$ & Martín & 1 & Baja \\
\hline Castelserás & $\mathrm{T}$ & Guadalope & 1 & Baja \\
\hline Castiello de Jaca & $\mathrm{H}$ & Barranco Especorral & 1 & Media \\
\hline Castigaleu & $\mathrm{H}$ & Cajigar & 1 & Baja \\
\hline Castiliscar & Z & Arroyo de Castiliscar & 1 & Media \\
\hline Castillazuelo & $\mathrm{H}$ & Vero & 1 & Baja \\
\hline Castillo Pompién & $\mathrm{H}$ & Isuela & 1 & Baja \\
\hline Castralvo & $\mathrm{T}$ & Barrancos & 1 & Media \\
\hline Cedrillas & $\mathrm{T}$ & Mijares & 1 & Media \\
\hline Celadas & $\mathrm{T}$ & Rambla del Tormo & 1 & Media \\
\hline Cella & $\mathrm{T}$ & Rambla de la Hoz & 1 & Baja \\
\hline Cetina & Z & Barranco de San Lázaro & 1 & Media \\
\hline Cimballa & Z & Piedra & 1 & Baja \\
\hline Ciscar & $\mathrm{H}$ & Cajigar & 1 & Baja \\
\hline Codos & Z & Gueimi, Grío & 2 & Media \\
\hline Conchel & $\mathrm{H}$ & Cinca & 1 & Media \\
\hline Cosa & $\mathrm{T}$ & Cosa, Barranco de Fuendelamadre & 2 & Media \\
\hline Cosuenda & Z & Barranco de Cosuenda & 1 & Alta \\
\hline Cuarte de Huerva & Z & Huerva & 1 & Alta \\
\hline Cubla & $\mathrm{T}$ & Barranco Horcajo & 1 & Baja \\
\hline Cuevas Labradas & $\mathrm{T}$ & Alfambra, Barranco de Corbalán & 2 & Media \\
\hline
\end{tabular}




\begin{tabular}{|c|c|c|c|c|}
\hline Entidad de población & Provincia & Curso fluvial & $N^{o}$ cursos & Densidad \\
\hline Daroca & Z & Arroyo de la Mina, Jiloca & 2 & Alta \\
\hline Ejea de los Caballeros & Z & Arba de Luesia, Arba de Biel & 2 & Alta \\
\hline El Burgo de Ebro & Z & Ebro & 1 & Alta \\
\hline El Campillo & $\mathrm{Z}$ & Gállego & 1 & Alta \\
\hline El Pobo & $\mathrm{T}$ & Barranco de Quiñonería & 1 & Media \\
\hline El Puente de Sabiñánigo & $\mathrm{H}$ & Gállego & 1 & Media \\
\hline El Run & $\mathrm{H}$ & Ésera & 1 & Baja \\
\hline El Sabinar & Z & Arba de Riguel & 1 & Baja \\
\hline El Soler & $\mathrm{H}$ & Isábena & 1 & Baja \\
\hline El Villarejo & $\mathrm{T}$ & Arroyo de Villarejo & 1 & Baja \\
\hline Embid de Ariza & Z & Henar o Deza & 1 & Media \\
\hline Embid de la Ribera & $\mathrm{Z}$ & Jalón & 1 & Baja \\
\hline Embún & $\mathrm{H}$ & Subordán & 1 & Baja \\
\hline Encinacorba & $Z$ & Frasno & 1 & Baja \\
\hline Epila & $Z$ & Jalón & 1 & Media \\
\hline Erés & $\mathrm{H}$ & Gállego & 1 & Baja \\
\hline Eriste & $\mathrm{H}$ & Eriste, Ésera & 2 & Media \\
\hline Erla & Z & Arba de Biel & 1 & Media \\
\hline Escalona & $\mathrm{H}$ & Vellós, Cinca & 2 & Media \\
\hline Escuer & $\mathrm{H}$ & Barranco & 1 & Media \\
\hline Estet & $\mathrm{H}$ & Barranco de Estet & 1 & Baja \\
\hline Fabara & $Z$ & Matarraña & 1 & Baja \\
\hline Fago & $\mathrm{H}$ & Majones & 1 & Baja \\
\hline Farasdués & Z & Farasdués & 1 & Media \\
\hline Farlete & Z & Barranco de San Caprasio & 1 & Alta \\
\hline Ferreruela de Huerva & $\mathrm{T}$ & Huerva & 1 & Baja \\
\hline Fiscal & $\mathrm{H}$ & Ara, Barranco Salvador & 2 & Alta \\
\hline Forcat & $\mathrm{H}$ & Noguera Ribagorzana & 1 & Baja \\
\hline Formiche Alto & $\mathrm{T}$ & Mijares & 1 & Baja \\
\hline Fortanete & $\mathrm{T}$ & Fortanete & 1 & Baja \\
\hline Fraga & $\mathrm{H}$ & Cinca & 1 & Alta \\
\hline Fuentes de Ebro & $\mathrm{Z}$ & Ginel & 1 & Alta \\
\hline Fuentes-Claras & $\mathrm{T}$ & Jiloca & 1 & Baja \\
\hline Fuentespalda & $\mathrm{T}$ & Barranco del Molino del Aceite & 1 & Baja \\
\hline Gallur & Z & Ebro & 1 & Media \\
\hline Gea de Albarracín & $\mathrm{T}$ & $\begin{array}{l}\text { Rambla de la Cruz del Alto, } \\
\text { Guadalaviar o Turia }\end{array}$ & 2 & Media \\
\hline Gelsa & Z & Ebro & 1 & Media \\
\hline Ginaste & $\mathrm{H}$ & Noguera Ribagorzana & 1 & Baja \\
\hline Gotor & $Z$ & Aranda & 1 & Baja \\
\hline Granja de San Pedro & Z & Jalón & 1 & Baja \\
\hline Grañén & $\mathrm{H}$ & Flumen & 1 & Media \\
\hline Graus & $\mathrm{H}$ & Ésera, Isábena & 2 & Media \\
\hline Grisén & Z & 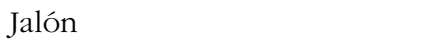 & 1 & Baja \\
\hline Guadalaviar & $\mathrm{T}$ & Guadalaviar o Turia & 1 & Media \\
\hline
\end{tabular}




\begin{tabular}{|c|c|c|c|c|}
\hline Entidad de población & Provincia & Curso fluvial & $N^{o}$ cursos & Densidad \\
\hline Gurrea de Gállego & $\mathrm{H}$ & Sotón & 1 & Baja \\
\hline Hecho & $\mathrm{H}$ & Aragón Subordán, Barranco & & \\
\hline & & de la Fuente & 2 & Alta \\
\hline Herrera de los Navarros & Z & Herrera & 1 & Alta \\
\hline Hijar & $\mathrm{T}$ & Martín & 1 & Baja \\
\hline Hospital & $\mathrm{H}$ & Cinca, Yoga & 2 & Baja \\
\hline Hostal de Ipiés & $\mathrm{H}$ & Gállego & 1 & Baja \\
\hline Huesca & $\mathrm{H}$ & Isuela & 1 & Alta \\
\hline Illueca & Z & Aranda, Barranco & 2 & Alta \\
\hline Jabaloyas & $\mathrm{T}$ & Barranco de la Canaleja & 1 & Baja \\
\hline Jaca & $\mathrm{H}$ & Aragón, Gas & 2 & Media \\
\hline Jaraba & Z & Mesa, Barranco de San Vicente & 2 & Media \\
\hline Jarque & Z & Aranda & 1 & Media \\
\hline Jorcas & $\mathrm{T}$ & Barranco del Regajo & 1 & Media \\
\hline Josa & $\mathrm{T}$ & Río de la Cantolera & 1 & Baja \\
\hline Juslibol & Z & Ebro & 1 & Baja \\
\hline La Almunia de & & & & \\
\hline Doña Godina & $\mathrm{Z}$ & Mediano o Alpartir & 1 & Alta \\
\hline La Escaleruela & $\mathrm{T}$ & Barranco de los Judíos & 1 & Baja \\
\hline La Hoz de la Vieja & $\mathrm{T}$ & Río de la Cantolera, Bco. 1, Bco. 2 & 3 & Media \\
\hline La Iglesuela del Cid & $\mathrm{T}$ & Barranco del Canto & 1 & Media \\
\hline La Paúl & $\mathrm{H}$ & Bco. La Paúl & 1 & Alta \\
\hline La Puebla de Roda & $\mathrm{H}$ & Isábena & 1 & Media \\
\hline La Virgen de la Vega & $\mathrm{T}$ & Alcalá o Balbona, Rambla & 2 & Baja \\
\hline La Zaida & Z & Aguas Vivas & 1 & Media \\
\hline Labuerda & $\mathrm{H}$ & Barranco de la Sierra & 1 & Alta \\
\hline Lafortunada & $\mathrm{H}$ & Cinca & 1 & Alta \\
\hline Lagueruela & $\mathrm{T}$ & Huerva & 1 & Baja \\
\hline Lalueza & $\mathrm{H}$ & Flumen & 1 & Baja \\
\hline Las Herrerías & $\mathrm{H}$ & Isábena & 1 & Baja \\
\hline Las Parras de Castellote & $\mathrm{T}$ & Barranco de las Parras & 1 & Media \\
\hline Las Pedrosas & Z & Barranco del Pueblo & 1 & Media \\
\hline Lascuarre & $\mathrm{H}$ & Barranco del Oliviadre & 1 & Media \\
\hline Lastiesas Baja & $\mathrm{H}$ & Barranco del Pinar & 1 & Baja \\
\hline Latre & $\mathrm{H}$ & Barranco Petral & 1 & Baja \\
\hline Lechago & $\mathrm{T}$ & Barranco de Cuencabuena & 1 & Media \\
\hline Letux & Z & Aguas Vivas & 1 & Media \\
\hline Libros & $\mathrm{T}$ & Guadalaviar o Turia & 1 & Media \\
\hline Ligüerre de Ara & $\mathrm{H}$ & Barranco de Toro & 1 & Media \\
\hline Llumes & $\mathrm{Z}$ & Piedra & 1 & Media \\
\hline Longares & $\mathrm{Z}$ & Barranco de la Gabardosa & 1 & Media \\
\hline Longás & $\mathrm{Z}$ & Onsella & 1 & Media \\
\hline Los Fayos & $\mathrm{Z}$ & Queiles & 1 & Media \\
\hline Los Huertos & $\mathrm{Z}$ & Ebro & 1 & Baja \\
\hline Loscos & $\mathrm{T}$ & Pilero & 1 & Baja \\
\hline
\end{tabular}




\begin{tabular}{|c|c|c|c|c|}
\hline Entidad de población & Provincia & Curso fluvial & $N^{o}$ cursos & Densidad \\
\hline Luceni & $Z$ & Ebro & 1 & Alta \\
\hline Luesia & Z & Barranco Villa & 1 & Media \\
\hline Lumpiaque & Z & Barranco de Rané & 1 & Media \\
\hline Maella & Z & Matarraña & 1 & Baja \\
\hline Magallón & Z & Huecha & 1 & Baja \\
\hline Maicas & $\mathrm{T}$ & Barranco Cavadera & 1 & Baja \\
\hline Malanquilla & Z & Ribota & 1 & Media \\
\hline Maluenda & Z & Jiloca & 1 & Media \\
\hline Manchones & $Z$ & Jiloca & 1 & Baja \\
\hline Manzanera & $\mathrm{T}$ & $\begin{array}{l}\text { Albentosa, Bco. de la Salada, } \\
\text { Bco. de las Vacas }\end{array}$ & 3 & Media \\
\hline Margudgued & $\mathrm{H}$ & Ara & 1 & Baja \\
\hline María de Huerva & $\mathrm{Z}$ & Huerva & 1 & Alta \\
\hline Martín del Río & $\mathrm{T}$ & Martín & 1 & Baja \\
\hline Mas de la Cabrera & $\mathrm{T}$ & Rambla de Pinilla & 1 & Baja \\
\hline Mases y Tamboril & $\mathrm{T}$ & Barranco del Barruezo & 1 & Baja \\
\hline Mequinenza & $\mathrm{Z}$ & Cinca & 1 & Media \\
\hline Miravete de la Sierra & $\mathrm{T}$ & Guadalope & 1 & Baja \\
\hline Molinos & $\mathrm{T}$ & Barranco de Valdepuertas & 1 & Baja \\
\hline Moneva & $\mathrm{Z}$ & Barranco del Tollo & 1 & Baja \\
\hline Monreal del Campo & $\mathrm{T}$ & Rambla de Palomarejos, Jiloca & 2 & Alta \\
\hline Montalbán & $\mathrm{T}$ & $\begin{array}{l}\text { Barranco del Infierno, Martín, } \\
\text { Barranco } 2\end{array}$ & 3 & Alta \\
\hline Montañana & $\mathrm{H}$ & San Juan & 1 & Baja \\
\hline Monterde de Albarracín & $\mathrm{T}$ & $\begin{array}{l}\text { Bco. de la Muela, } \\
\text { Rambla de Monterde }\end{array}$ & 2 & Alta \\
\hline Monzalbarba & Z & Ebro & 1 & Media \\
\hline Monzón & $\mathrm{H}$ & Cinca, Sosa & 2 & Alta \\
\hline Mora de Rubielos & $\mathrm{T}$ & $\begin{array}{l}\text { Barranco del Mar de Perales, } \\
\text { Moral }\end{array}$ & 2 & Alta \\
\hline Morata de Jiloca & Z & Jiloca & 1 & Baja \\
\hline Mores & Z & Jalón & 1 & Media \\
\hline Morillo de Liena & $\mathrm{H}$ & Ésera, Barranco de Bacamonta & 2 & Baja \\
\hline Mosqueruela & $\mathrm{T}$ & Barranco de la Horca & 1 & Media \\
\hline Movera & Z & Ebro & 1 & Baja \\
\hline Moyuela & Z & Moyuela & 1 & Media \\
\hline Mozota & Z & Huerva & 1 & Media \\
\hline Muel & Z & Huerva & 1 & Media \\
\hline Munébrega & Z & $\begin{array}{l}\text { Rambla del Molino, } \\
\text { Barranco del olivo }\end{array}$ & 2 & Baja \\
\hline Muniesa & $\mathrm{T}$ & Reguero Granjera & 1 & Baja \\
\hline Murero & $\mathrm{Z}$ & Jiloca & 1 & Baja \\
\hline Naval & $\mathrm{H}$ & Naval & 1 & Baja \\
\hline Navardún & $\mathrm{Z}$ & Onsella & 1 & Media \\
\hline Navarrete del Río & $\mathrm{T}$ & Barranco de la Peña & 1 & Media \\
\hline
\end{tabular}




\begin{tabular}{|c|c|c|c|c|}
\hline Entidad de población & Provincia & Curso fluvial & $N^{o}$ cursos & Densidad \\
\hline Navarri & $\mathrm{H}$ & Ésera, Barranco de Foradada & 2 & Baja \\
\hline Nigüella & Z & Isuela & 1 & Baja \\
\hline Noales & $\mathrm{H}$ & Baliera & 1 & Baja \\
\hline Nocito & $\mathrm{H}$ & Guatizalema & 1 & Baja \\
\hline Noguera & $\mathrm{T}$ & Noguera & 1 & Media \\
\hline Nogueruelas & $\mathrm{T}$ & Barranco & 1 & Media \\
\hline Nonaspe & Z & Matarraña & 1 & Baja \\
\hline Novillas & Z & Ebro & 1 & Alta \\
\hline Nuez de Ebro & Z & Barranco de Nuez & 1 & Alta \\
\hline Obón & $\mathrm{T}$ & Barranco Santa María & 1 & Media \\
\hline Oliete & $\mathrm{T}$ & Martín & 1 & Media \\
\hline Orera & Z & Rambla de Orera & 1 & Media \\
\hline Orihuela del Tremedal & $\mathrm{T}$ & Gallo, Barranco de Valdecalera & 2 & Media \\
\hline Orós Bajo & $\mathrm{H}$ & Barranco de Lucas & 1 & Baja \\
\hline Pancrudo & $\mathrm{T}$ & Barranco & 1 & Baja \\
\hline Paniza & Z & Arroyo de Carracariñena & 1 & Media \\
\hline Panticosa & $\mathrm{H}$ & Caldarés, Bolatica & 2 & Media \\
\hline Paracuellos de Jiloca & $\mathrm{Z}$ & Jiloca & 1 & Baja \\
\hline Paracuellos de la Ribera & Z & Jalón, Barranco del Val & 2 & Baja \\
\hline Parzán & $\mathrm{H}$ & Barrosa & 1 & Baja \\
\hline Pastriz & $\mathrm{Z}$ & Ebro & 1 & Media \\
\hline Peñaflor & Z & Gállego & 1 & Media \\
\hline Peñalba & $\mathrm{H}$ & Barranco del Reguero & 1 & Alta \\
\hline Peñarroyas & $\mathrm{T}$ & Martín & 1 & Baja \\
\hline Peracense & $\mathrm{T}$ & Arroyo de Valdelafuente & 1 & Baja \\
\hline Peralejos & $\mathrm{T}$ & Alfambra & 1 & Baja \\
\hline Peralta de la Sal & $\mathrm{H}$ & Calasanz & 1 & Media \\
\hline Perarrúa & $\mathrm{H}$ & Ésera & 1 & Media \\
\hline Pertusa & $\mathrm{H}$ & Alcanadre & 1 & Baja \\
\hline Piedrahita & $\mathrm{T}$ & Arroyo de la Cañada & 1 & Baja \\
\hline Pina de Ebro & Z & Ebro & 1 & Media \\
\hline Pitarque & $\mathrm{T}$ & Barranco & 1 & Alta \\
\hline Plan & $\mathrm{H}$ & Barranco de Foricón & 1 & Alta \\
\hline Plasencia de Jalón & Z & Jalón & 1 & Media \\
\hline Pleitas & $\mathrm{Z}$ & Jalón & 1 & Baja \\
\hline Poblado Monsanto & $\mathrm{H}$ & Cinca & 1 & Media \\
\hline Poleñino & $\mathrm{H}$ & Flumen & 1 & Media \\
\hline Pomar de Cinca & $\mathrm{H}$ & Barranco La Clamor & 1 & Baja \\
\hline Pompenillo & $\mathrm{H}$ & Isuela & 1 & Baja \\
\hline Pozán de Vero & $\mathrm{H}$ & Vero & 1 & Media \\
\hline Pozuel de Ariza & $\mathrm{Z}$ & Nágima & 1 & Baja \\
\hline Pradilla de Ebro & $\mathrm{Z}$ & Ebro & 1 & Alta \\
\hline Puebla de Alfindén & $\mathrm{Z}$ & Barranco de las Casas & 1 & Alta \\
\hline Puendeluna & $\mathrm{Z}$ & Gállego & 1 & Media \\
\hline Puente de Montañana & $\mathrm{H}$ & Noguera Ribagorzana & 1 & Media \\
\hline
\end{tabular}




\begin{tabular}{|c|c|c|c|c|}
\hline Entidad de población & Provincia & Curso fluvial & $N^{\circ}$ cursos & Densidad \\
\hline Puerto de Astún & $\mathrm{H}$ & Aragón & 1 & Baja \\
\hline Quinto & Z & Bco.Val1, Bc.Val2 & 2 & Baja \\
\hline Remolinos & Z & Ebro, Barranco & 2 & Baja \\
\hline Ricla & Z & Jalón & 1 & Alta \\
\hline Rivas & Z & Arba de Luesia & 1 & Media \\
\hline Rubielos de Mora & $\mathrm{T}$ & Rubielos & 1 & Baja \\
\hline Rueda de Jalón & Z & Jalón & 1 & Media \\
\hline Sabiñánigo & $\mathrm{H}$ & Río de la Tulipana, Gállego, Aurín & 3 & Alta \\
\hline Sádaba & Z & Arba de Riguel & 1 & Alta \\
\hline Sahún & $\mathrm{H}$ & Barranco de Cambra & 1 & Baja \\
\hline Salinas & $\mathrm{H}$ & Cinca & 1 & Baja \\
\hline Sallent de Gállego & $\mathrm{H}$ & Aguas Limpias, Gállego & 2 & Alta \\
\hline Salvatierra de Esca & $\mathrm{Z}$ & Esca & 1 & Baja \\
\hline San Blas & $\mathrm{T}$ & Rambla San blas & 1 & Baja \\
\hline San Juan de Mozarrifar & Z & Gállego & 1 & Media \\
\hline San Juan de Plan & $\mathrm{H}$ & Cinqueta & 1 & Media \\
\hline San Martín del Río & $\mathrm{T}$ & Jiloca & 1 & Baja \\
\hline San Mateo de Gállego & Z & Barranco el Barranco & 2 & Media \\
\hline Sangarrén & $\mathrm{H}$ & Flumen & 1 & Baja \\
\hline Santa Bárbara & $\mathrm{T}$ & Aliaga & 1 & Media \\
\hline Santa Cilia de Jaca & $\mathrm{H}$ & Aragón & 1 & Alta \\
\hline Santa Cruz de la Serós & $\mathrm{H}$ & Barranco Carbonera & 1 & Baja \\
\hline Santa Eulalia & Z & Rambla de los Raboseros & 1 & Media \\
\hline Santa Liestra & & & & \\
\hline y San Quílez & $\mathrm{H}$ & Barranco de Gabarros & 1 & Baja \\
\hline Santed & $\mathrm{Z}$ & Arroyo de la Aldeuela & 1 & Media \\
\hline Sarvisé & $\mathrm{H}$ & Barranco de Buesa & 1 & Baja \\
\hline Saviñán & $\mathrm{Z}$ & Jalón & 1 & Media \\
\hline Seira & $\mathrm{H}$ & Ésera & 1 & Baja \\
\hline Serraduy & $\mathrm{H}$ & Isábena & 1 & Media \\
\hline Sobradiel & Z & Ebro & 1 & Media \\
\hline Talamantes & Z & Barranco de Valdeherrera & 1 & Media \\
\hline Tarazona & Z & Queiles & 1 & Alta \\
\hline Tauste & Z & Barranco Santa Bárbara, Arba & 2 & Alta \\
\hline Terrer & Z & Barranco del Salto del Plano & & \\
\hline & & Cardiel & 1 & Alta \\
\hline Teruel & $\mathrm{T}$ & Guadalaviar o Turia, & & \\
\hline & & Rambla San Julián & 2 & Media \\
\hline Tierrantona & $\mathrm{H}$ & Barranco Usía & 1 & Media \\
\hline Tobed & $\mathrm{Z}$ & Grío, Barranco de Valdeolivo & 2 & Media \\
\hline Torla & $\mathrm{H}$ & Barranco Cueto & 1 & Alta \\
\hline Torre los Negros & $\mathrm{T}$ & Barranco de la Covacha & 1 & Media \\
\hline Torrehermosa & $\mathrm{Z}$ & Arroyo de la Cañada & 1 & Baja \\
\hline Torrente de Cinca & $\mathrm{H}$ & Cinca & 1 & Baja \\
\hline Torres de Albarracín & $\mathrm{T}$ & Barranco de los Borricos & 1 & Baja \\
\hline
\end{tabular}




\begin{tabular}{|c|c|c|c|c|}
\hline Entidad de población & Provincia & Curso fluvial & $N^{o}$ cursos & Densidad \\
\hline Torres de Barbués & $\mathrm{H}$ & Flumen & 1 & Baja \\
\hline Torres de Berrellén & Z & Ebro & 1 & Alta \\
\hline Torrijo de la Cañada & $\mathrm{Z}$ & $\begin{array}{l}\text { Manubles, Barranco de Val } \\
\text { de la Casa }\end{array}$ & 2 & Alta \\
\hline Torrijo del Campo & $\mathrm{T}$ & Jiloca & 1 & Media \\
\hline Tortajada & $\mathrm{T}$ & Alfambra & 1 & Baja \\
\hline Tosos & Z & Huerva & 1 & Media \\
\hline Trasobares & Z & Isuela, Bco. de Viñalajuén & 2 & Baja \\
\hline Uncastillo & Z & Arba de Riguel, Barranco de & & \\
\hline Urdués & $\mathrm{H}$ & $\begin{array}{l}\text { Baldelavina } \\
\text { Barranco de Romaciete, } \\
\text { Barranco Fayanás }\end{array}$ & 2 & Alta \\
\hline Urrea de Jalón & $\mathrm{Z}$ & 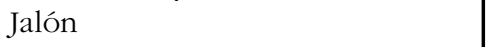 & 1 & Media \\
\hline Utebo & Z & Ebro & 1 & Alta \\
\hline Utrillas & $\mathrm{T}$ & Barranco 1, Barranco 2 & 2 & Media \\
\hline Valacloche & $\mathrm{T}$ & Camarena & 1 & Baja \\
\hline Valbona & $\mathrm{T}$ & Balbona & 1 & Alta \\
\hline Valdeltormo & $\mathrm{T}$ & Barranco Chulo & 1 & Baja \\
\hline Valderrobres & $\mathrm{T}$ & Matarraña & 1 & Alta \\
\hline Valpalmas & $\mathrm{Z}$ & Barranco la Barluenga & 1 & Baja \\
\hline Velilla de Cinca & $\mathrm{H}$ & Barranco & 1 & Media \\
\hline Velilla de Ebro & $\mathrm{Z}$ & Barranco de la Cerrada, Ebro & 2 & Media \\
\hline Velilla de Jiloca & $\mathrm{Z}$ & Barranco de Pueblo & 1 & Media \\
\hline Villafeliche & $\mathrm{Z}$ & Jiloca & 1 & Baja \\
\hline Villafranca de Ebro & $\mathrm{Z}$ & Barranco de Villafranca de Ebro & 1 & Alta \\
\hline Villafranca del Campo & $\mathrm{T}$ & Jiloca & 1 & Media \\
\hline Villalangua & $\mathrm{H}$ & Asabón & 1 & Media \\
\hline Villalba Baja & $\mathrm{T}$ & Alfambra, Barranco de la Cordillera & 2 & Media \\
\hline Villanúa & $\mathrm{H}$ & $\begin{array}{l}\text { Bco. Lierde, Aragón, Barranco } \\
\text { de Villanúa }\end{array}$ & 3 & Alta \\
\hline Villanueva de Huerva & Z & Huerva & 1 & Baja \\
\hline Villar de los Navarros & Z & Cámaras & 1 & Media \\
\hline Villar del Cobo & $\mathrm{T}$ & Griegos, Guadalaviar o Turia & 2 & Alta \\
\hline Villarroya de la Sierra & $\mathrm{Z}$ & Ribota & 1 & Media \\
\hline Villarroya de los Pinares & $\mathrm{T}$ & Guadalope & 1 & Baja \\
\hline Villaspesa & $\mathrm{T}$ & Rambla de Villaespesa & 1 & Alta \\
\hline Villastar & $\mathrm{T}$ & Barranco 1, Barranco 2 & 2 & Baja \\
\hline Villel & $\mathrm{T}$ & $\begin{array}{l}\text { Rambla Chartera, Guadalaviar } \\
\text { o Turia }\end{array}$ & 2 & Baja \\
\hline Vinaceite & $\mathrm{T}$ & Aguas Vivas & 1 & Baja \\
\hline Visiedo & $\mathrm{T}$ & Rambla de la Corredera & 1 & Alta \\
\hline Vistabella & $\mathrm{Z}$ & Huerva & 1 & Baja \\
\hline Vivel del Río Martín & $\mathrm{T}$ & Segura & 1 & Baja \\
\hline Zaragoza & $\mathrm{Z}$ & Ebro, Huerva, Gállego & 3 & Alta \\
\hline Zuera & Z & Gállego & 1 & Media \\
\hline
\end{tabular}


Figura 1. Confluencia del río Jalón y el Manubles en Ateca (Zaragoza). Foto: Sistema de Información Territorial de Aragón (SITAR). Gobierno de Aragón.

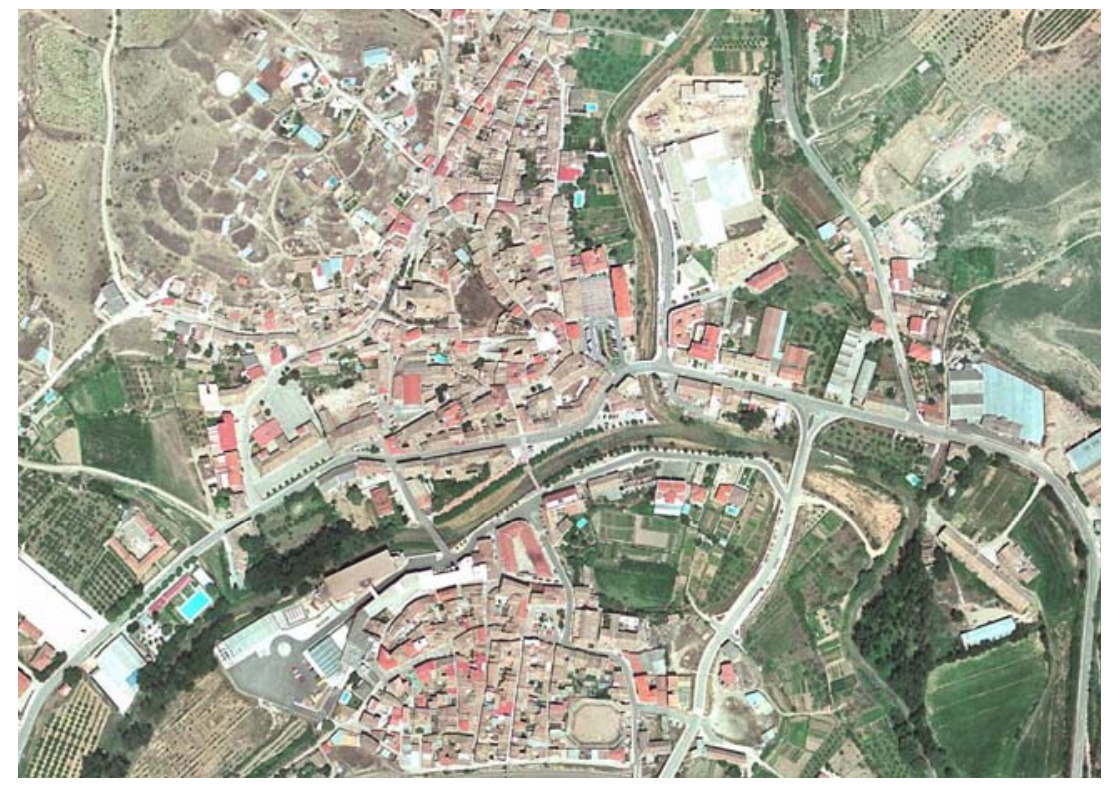


Figura 2. Barranco Cuatro Esquinas (Cariñena, Zaragoza). Foto.

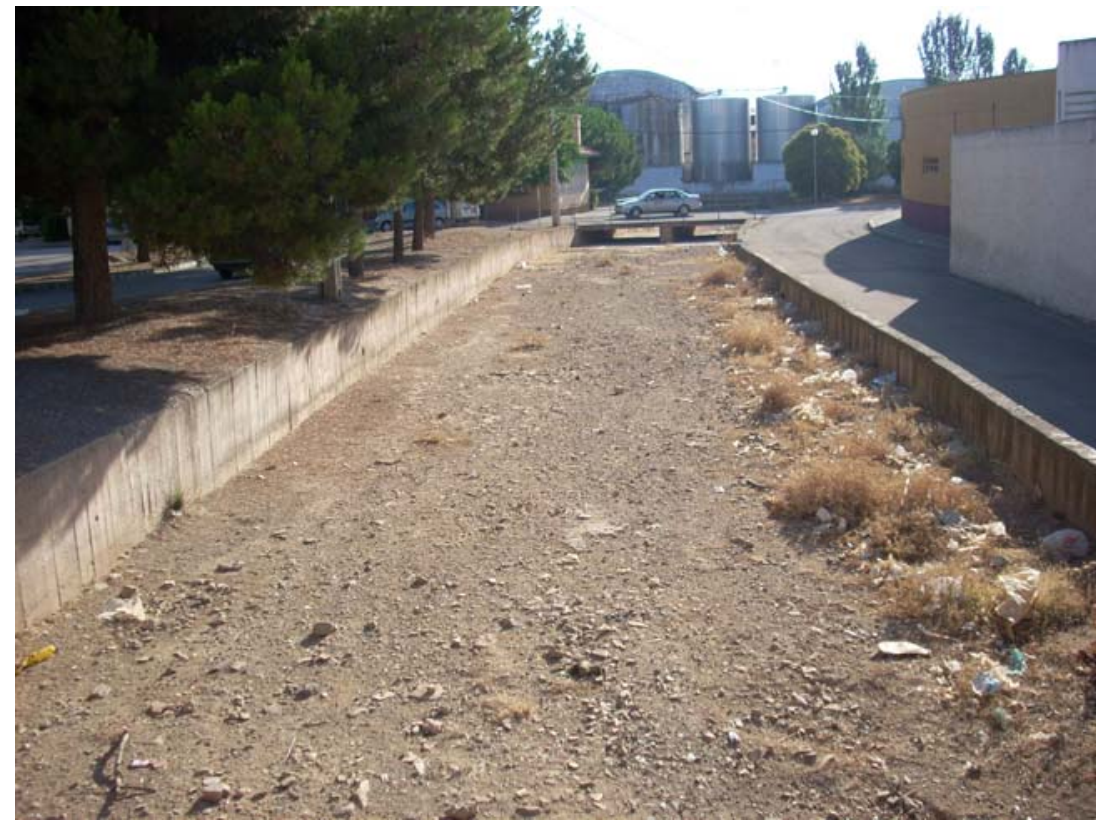


Figura 4. Zona inundable por un río de llanura: el Cinca en Fraga (Huesca). Foto.

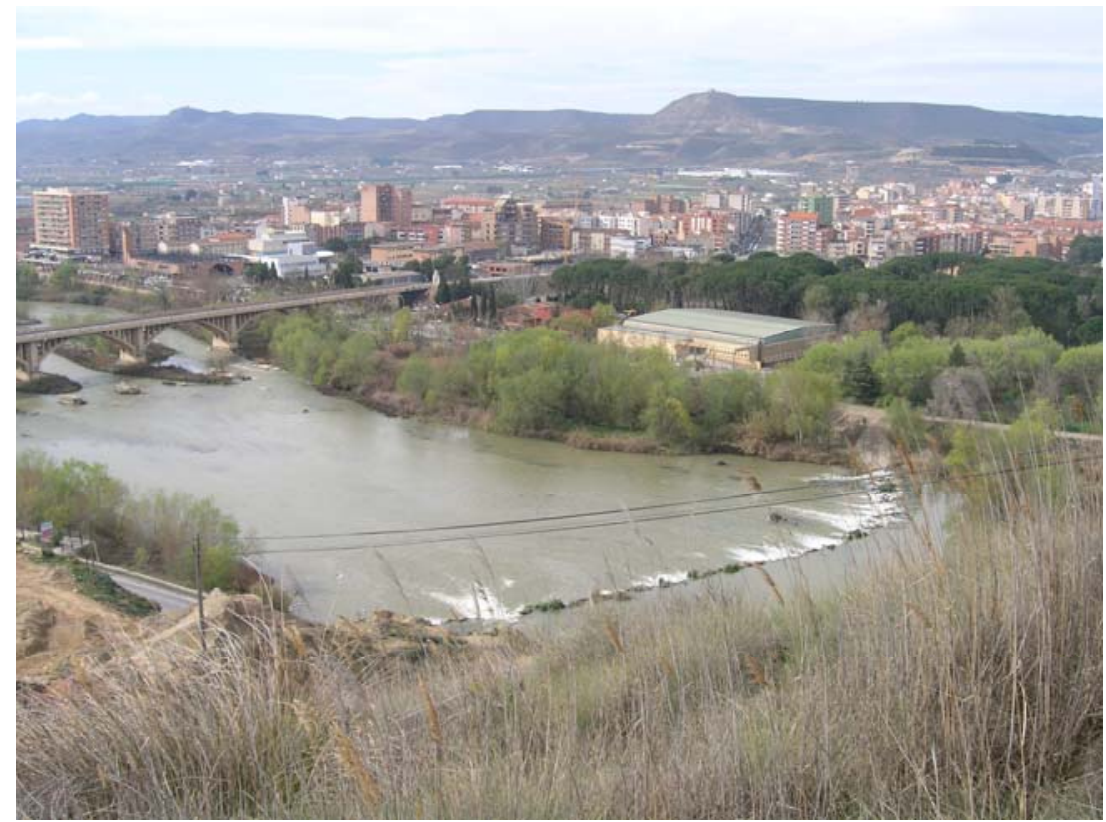


Figura 5. Cono de deyección del barranco Lierde (Villanúa, Huesca). Foto.

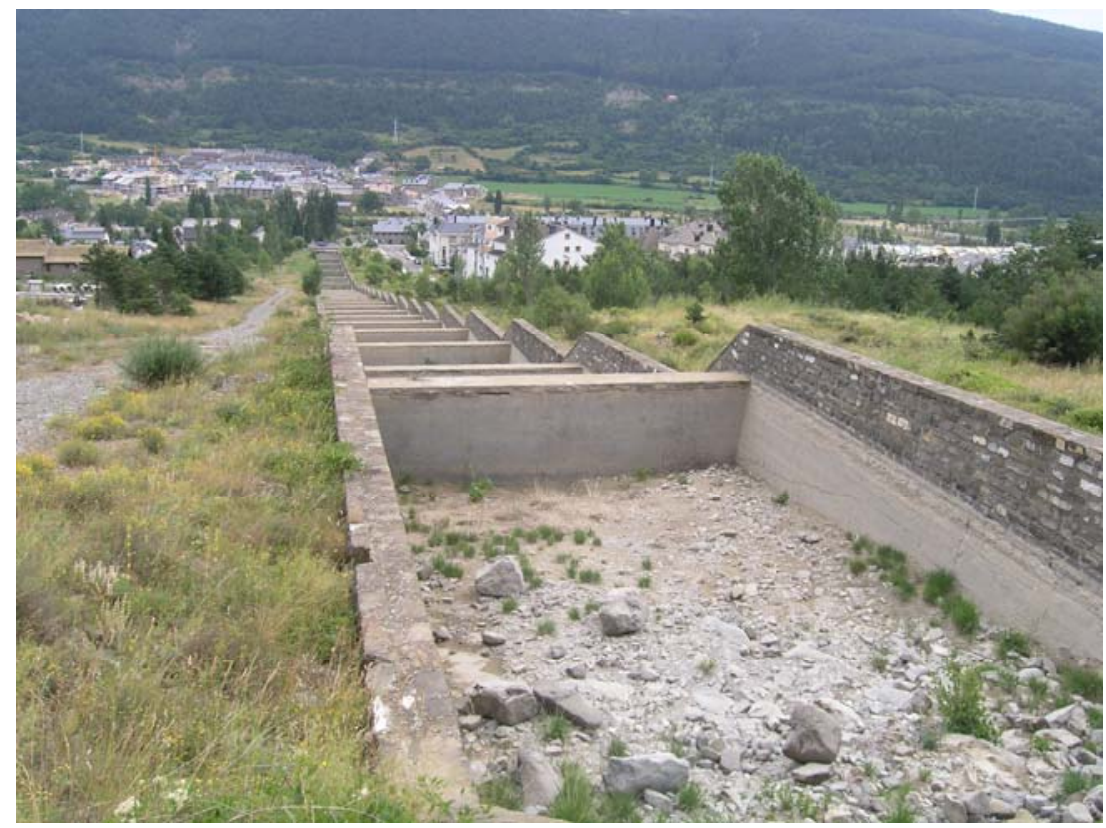

